\title{
Predictable Dynamics of Opinion Forming for Networks With Antagonistic Interactions
}

\author{
Claudio Altafini and Gabriele Lini \\ Journal Article
}

\section{Tweet}

N.B.: When citing this work, cite the original article.

C2016 IEEE. Personal use of this material is permitted. However, permission to reprint/republish this material for advertising or promotional purposes or for creating new collective works for resale or redistribution to servers or lists, or to reuse any copyrighted component of this work in other works must be obtained from the IEEE.

Claudio Altafini and Gabriele Lini, Predictable Dynamics of Opinion Forming for Networks With Antagonistic Interactions, IEEE Transactions on Automatic Control, 2015. 60(2), pp.342357.

http://dx.doi.org/10.1109/TAC.2014.2343371

Postprint available at: Linköping University Electronic Press

http://urn.kb.se/resolve?urn=urn:nbn:se:liu:diva-114571

\section{I.U. UNWENRSGS}




\title{
Predictable dynamics of opinion forming for networks with antagonistic interactions
}

\author{
Claudio Altafini and Gabriele Lini
}

\begin{abstract}
For communities of agents which are not necessarily cooperating, distributed processes of opinion forming are naturally represented by signed graphs, with positive edges representing friendly and cooperative interactions and negative edges the corresponding antagonistic counterpart. Unlike for nonnegative graphs, the outcome of a dynamical system evolving on a signed graph is not obvious and it is in general difficult to characterize, even when the dynamics are linear. In this paper we identify a significant class of signed graphs for which the linear dynamics are however predictable and show many analogies with positive dynamical systems. These cases correspond to adjacency matrices that are eventually positive, for which the PerronFrobenius property still holds and implies the existence of an invariant cone contained inside the positive orthant. As examples of applications, we determine cases in which it is possible to anticipate or impose unanimity of opinion in decision/voting processes even in presence of stubborn agents, and show how it is possible to extend the PageRank algorithm to include negative links.
\end{abstract}

Index terms - Opinion dynamics; Signed graphs; Eventually positive matrices; Perron-Frobenius theorem; Invariant Cones; Social networks.

\section{INTRODUCTION}

A popular trend in the literature on networked control systems is the study of distributed dynamical models of opinion forming on "social networks", intended as communities of interacting and reciprocally influencing agents [1], [2], [8], [21], [22], [30], [46]. An implicit assumption in this literature is that the agents collaborate to achieve a common goal. This is however a limitation in many settings potentially of interest. Think for example of contexts in which two or more groups of agents compete with each other, like for instance in models of competing business cartels, or in team sports, or in a resource allocation scheme. More generally, think of social networks in which each agent has a pattern of positive/negative relationships with other agents, representing alliance/rivalry, cooperation/competition, trust/distrust, etc. All these cases lead to non-collaborative frameworks not captured by the models conventionally used. In particular, if collaboration is encoded as nonnegativity of the adjacency matrix of the graph,

C. Altafini is with SISSA, International School for Advanced Studies, via Bonomea 265, I-34136 Trieste, Italy, and with the Division of Automatic Control, Dept. of Electrical Engineering, Linköping University, SE-58183, Sweden. email: claudio.altafini@liu.se

G. Lini was with SISSA, International School for Advanced Studies, Trieste, Italy. $\mathrm{He}$ is now with Magneti Marelli S.p.A. Viale Carlo Emanuele II 150, 10078 Venaria Reale (TO), Italy. email: gabriele.lini@magnetimarelli.com

Work sponsored in part by a grant from the European Social Fund, through the SHARM project at SISSA.

A preliminary version of this work was presented at the European Control Conference, June 2014. including non-collaborative interactions means resorting to signed graphs [3], [16], [18], [38], [44], [45].

Assume our signed graphs are a tool through which a community of agents expresses opinions on a subject. The "opinion" could be intended as a vote on a subject, a decision making process, a measure of reputation, or even a ranking of the nodes. If the process of opinion forming is distributed, then each node has to use the interactions with its first neighbors to form its own opinion. Following [11], in this setting it is reasonable to assume that positive interactions correspond to positive influences in the opinion forming process, and negative interactions to negative influences. In [3] we have investigated what happens to the dynamics in the special case of signed graphs which are structurally balanced [4], i.e., that can be rendered nonnegative by a change of orthant order, like in a monotone system [39].

In this paper we are interested in going beyond structural balance, and understanding in which cases a dynamical system on a signed graph can achieve an unanimous opinion, intended as convergence to the first orthant of $\mathbb{R}^{n}$ (i.e., $\mathbb{R}_{+}^{n}$ ) or to its negation $\left(\mathbb{R}_{-}^{n}\right)$. For linear models, convergence to these two orthants is naturally associated to the Perron-Frobenius theorem, in which the eigenvector associated to the dominant eigenvalue (the spectral radius) is positive and hence all trajectories tend to align themselves along it. In fact, the Perron-Frobenius condition is for example at the basis of the literature on the consensus problem [28], [36], in which all agents are asked to converge to the same value, hence to a specific point in $\mathbb{R}_{+}^{n}$ or $\mathbb{R}_{-}^{n}$. Our concept of unanimity is more flexible and asks only for a consensus on the signs of the opinions, meaning that any state in $\mathbb{R}_{+}^{n}$ or $\mathbb{R}_{-}^{n}$ still represents an unanimous opinion (although some agents will be "more convinced" than others).

In classical linear algebra, the Perron-Frobenius theorem is formulated for entry-wise nonnegative matrices [5]. However, in recent times, it has been shown that a Perron-Frobenius condition holds also for a class of matrices having some negative entries, called eventually positive matrices [33], [17], [34]. We show in this paper that if a linear dynamics of opinion forming is eventually positive, then the system achieves unanimity. However, unlike for nonnegative adjacency matrices (or, more generally, for positive systems [20]), $\mathbb{R}_{+}^{n}$ is not invariant for the dynamics.

In order to distinguish the concept we are interested in this paper from the standard notion of orthant invariance, we introduce the concept of holdability [32]. An orthant is holdable if all trajectories are bound to enter it (or its negation) after a transient, and then stay there forever. In practice, orthant holdability is a form of "delayed" orthant invariance: there always exist nonnegative initial conditions which transiently 
exit $\mathbb{R}_{+}^{n}$, only to return in it at later times (and remain in it thereafter). We show in the paper that eventual positivity implies orthant holdability, and is equivalent to the existence of an invariant cone properly contained inside $\mathbb{R}_{+}^{n}$. The perspective of invariant linear systems is very useful to understand other, more subtle, situations such as what happens to the dynamics of opinion forming in presence of stubborn agents. Stubborn agents are nodes who can influence the other nodes but whose opinion in unchangeable [22], [1], [21]. Using the theory of constrained linear systems [7], the problem of preserving unanimity in presence of stubborn agents can be rephrased as that of (cone) invariance in presence of persistent constant disturbances [29].

A matrix is eventually positive if at a certain power it becomes positive and stays positive for all higher powers [26]. In discrete-time, this condition implies that a suitably downsampled version of an eventually positive system is positive, hence highlighting further the transient nature of the effects of the negative edges in these systems. The case in which the downsampled transition matrix is stochastic is particularly interesting, because it implies that for the original discrete-time system a probabilistic interpretation is lost, but only transiently.

The geometric perspective we develop for eventually positive adjacency matrices allows us to easily understand why the type of antagonism investigated in this paper is fundamentally different from the structural balance studied in [4]. While structurally balanced systems are orthant invariant with respect to one of the $2^{n}$ orthants of $\mathbb{R}^{n}$, eventually positive systems admit an invariant cone which is always sitting inside $\mathbb{R}_{+}^{n}$ but cannot coincide with the entire $\mathbb{R}_{+}^{n}$. A consequence of this fundamental difference is that while structural balance is a "qualitative property", i.e., it is common to all matrices having the same sign pattern [27], eventual positivity depends on the numerical values of the entries of an adjacency matrix, which makes it more difficult to deal with, especially in the context of distributed control synthesis.

Notice that it is also possible to combine the two types of antagonism. In fact, if holding to $\mathbb{R}_{+}^{n}$ means achieving an unanimous opinion, and corresponds to the existence of an invariant cone contained in $\mathbb{R}_{+}^{n}$, then a natural extension is an opinion which is holdable but not unanimous, i.e., a system whose trajectories converge to an invariant cone fully contained in one of the orthants of $\mathbb{R}^{n}$ (or in its negation). This broaden considerably the range of matrices for which the outcome of a process of opinion forming is predictable to basically all matrices possessing an invariant cone, a well studied problem in both linear algebra [5] and control theory [7], [12], [19], [43]. We call this a "signed" Perron-Frobenius condition.

Finally, although in the paper we adopt the terminology of "opinion forming" for a distributed system, the methodology is applicable also to any problem that can be formulated as a distributed linear dynamical system on a signed graph. For example one can replace "opinion forming" with "decision making" or with "voting scheme" or even with "ranking". As an alternative example of application, in fact, we show how it is possible to extend an algorithm like Google's PageRank
[9], [10] in order to cope with negative links. In this context, the entries of the adjacency matrix are hyperlinks, and it is known that a consistent fraction of links can in principle be classifiable as "negative" links (links from spamindexing, cloaking and other "black hat" search engine optimization practices). Several algorithms have already appeared in order to cope with them [14], [13], [42]. None of these approaches is similar to ours. A possible extension to negative ranking is also shown.

The rest of this paper is organized as follows: in Section II we review the linear-algebraic concepts needed later on and establish a relationship between eventually positive matrices and invariant cones; in Section III we investigate unanimity of opinion dynamics, possibly in presence of stubborn agents, while in Section IV we show how to design control laws that achieve unanimity. The case of non-unanimous opinions is treated in Section V. Finally in Section VI discrete-time opinion dynamics is discussed and the application to PageRank is developed in detail.

\section{LINEAR ALGEBRAIC PRELIMINARIES}

Given a matrix $A=\left(a_{i j}\right) \in \mathbb{R}^{n \times n}, A \geq 0$ means $a_{i j} \geq 0$ for any $i, j \in 1, \ldots, n$, and $A \neq 0$, while $A>0$ means $a_{i j}>0$ for all $i, j=1, \ldots, n$. The matrix $A$ is called nonnegative (resp. positive) if $A \geq 0$ (resp. $A>0$ ). This notation is used also for vectors. The spectrum of $A$ is denoted $\operatorname{sp}(A)=\left\{\lambda_{1}(A), \ldots, \lambda_{n}(A)\right\}$, where $\lambda_{i}(A), i=1, \ldots, n$, are the eigenvalues of $A$, and the vector space generated by its columns is $\operatorname{span}(A)$. The spectral radius of $A, \rho(A)$, is the smallest real positive number such that $\rho(A) \geq\left|\lambda_{i}(A)\right|$, $\forall i=1, \ldots, n$. A matrix $A \in \mathbb{R}^{n \times n}$ is said to be irreducible if there does not exist a permutation matrix $\Pi$ such that $\Pi^{T} A \Pi$ is block triangular, that is

$$
\Pi^{T} A \Pi \neq\left[\begin{array}{cc}
A_{11} & A_{12} \\
0 & A_{22}
\end{array}\right],
$$

where $A_{11}, A_{12}$ and $A_{22}$ are nontrivial square matrices. We say that $A$ is asymptotically stable if $\operatorname{Re}\left[\lambda_{i}(A)\right]<0$ for any $i$, and it is marginally stable if $\operatorname{Re}\left[\lambda_{i}(A)\right] \leq 0$ and $\lambda_{i}(A)$ such that $\operatorname{Re}\left[\lambda_{i}(A)\right]=0$ is a simple root of the minimal polynomial of $A$. The directed graph whose adjacency matrix is $A$ is indicated $\Gamma(A)$. It has an edge connecting the node $j$ to the node $i$ if and only if $a_{i j} \neq 0$. We indicate $\operatorname{adj}(i)$ the set of nodes adjacent to $i: j \in \operatorname{adj}(i)$ if and only if $a_{i j} \neq 0$. $\Gamma(A)$ is strongly connected if and only if $A$ is irreducible.

\section{A. Eventual positivity and Perron-Frobenius property}

Definition 1 A matrix $A \in \mathbb{R}^{n \times n}$ has the weak PerronFrobenius property if $\rho(A)$ is a positive eigenvalue of $A$ and $v_{r}$, the right eigenvector relative to $\rho(A)$, is nonnegative.

Definition 2 A matrix $A \in \mathbb{R}^{n \times n}$ has the strong PerronFrobenius property if $\rho(A)$ is a simple positive eigenvalue of $A$ s.t. $\rho(A)>|\lambda|$ for every $\lambda \in \operatorname{sp}(A), \lambda \neq \rho(A)$, and $v_{r}$, the right eigenvector relative to $\rho(A)$, is positive. 
Denote $\mathcal{P} \mathcal{F}_{n}$ (resp. $\mathcal{W} \mathcal{P} \mathcal{F}_{n}$ ) the set of matrices in $\mathbb{R}^{n \times n}$ that possess the strong (resp. weak) Perron-Frobenius property. Although these properties are naturally associated with nonnegative matrices, in recent times it has been shown that they hold also for matrices with some negative entries, in particular for the so-called eventually positive and eventually nonnegative matrices [23], [26], [33], [17].

Definition 3 A real square matrix $A$ is said to be eventually positive (resp. eventually nonnegative) if $\exists k_{o} \in \mathbb{N}$ such that $A^{k}>0$ (resp. nonnilpotent and $A^{k} \geq 0$ ) for all $k \geq k_{0}$.

The smallest integer $k_{o}$ of Definition 3 is called the power index of $A$. Following [34], eventually positive (resp. eventually nonnegative) matrices will be denoted $A>0$ (resp. $A \stackrel{\vee}{\geq} 0$ ). Clearly, $A \stackrel{\vee}{>} 0$ implies $A$ irreducible, while this is not necessarily true for $A \geq 0$. Eventually positive matrices are a subclass of the eventually nonnegative ones for which a necessary and sufficient condition for the fulfillment of the strong Perron-Frobenius property is available.

Theorem 1 ([33], Theorem 2.2) For $A \in \mathbb{R}^{n \times n}$ the following are equivalent:

1) Both $A, A^{T} \in \mathcal{P} \mathcal{F}_{n}$;

2) $A \stackrel{\vee}{>} 0$;

3) $A^{T}>0$

Example 1 The matrix

$$
A=\left[\begin{array}{cccc}
0 & 0 & 0 & 39 \\
0 & 0 & 92 & 9 \\
0 & 117 & 0 & -50 \\
5 & 0 & 111 & 0
\end{array}\right]
$$

is such that $A \in \mathcal{P} \mathcal{F}_{n}$ but $A^{T} \notin \mathcal{P} \mathcal{F}_{n}$. Therefore $A \not{\triangleright} \not 0$.

The eventual nonnegativity of (nonnilpotent) matrices is instead a sufficient (but not necessary) condition for the weak Perron-Frobenius property.

Theorem 2 ([33], Theorem 2.3) Given $A \in \mathbb{R}^{n \times n}$ nonnilpotent, if $A \geq 0$ then $A, A^{T} \in \mathcal{W} \mathcal{P} \mathcal{F}_{n}$.

Notice that from Theorem 1 of [26], we have an easy test of eventual positivity: $A$ is eventually positive iff $A^{k}>0$ and $A^{k+1}>0$ for some positive integer $k$.

Lemma 1 Consider $A \stackrel{\vee}{>} 0$ and denote $v_{r}>0$ its right eigenvector. Then any eigenvector $v_{1}$ of $A$ such that $v_{1}>0$ must be a multiple of $v_{r}$.

Proof. Assume $A \stackrel{\vee}{>} 0$ has two distinct eigenvectors $v_{r}>0$ and $v_{1}>0$ for which $v_{1} \neq \alpha v_{r}, \alpha \in \mathbb{R}$, i.e. $\exists \lambda_{1} \in \operatorname{sp}(A)$, $\lambda_{1} \neq \rho(A)$, such that $A v_{1}=\lambda_{1} v_{1}$. But then $\exists k_{o} \in \mathbb{N}$ such that for $k \geq k_{o}$ we have

$$
\begin{aligned}
& A^{k} v_{r}=\rho(A)^{k} v_{r} \\
& A^{k} v_{1}=\lambda_{1}^{k} v_{1}
\end{aligned}
$$

which is a contradiction since $A^{k}>0$ can have only one positive eigenvector (see e.g. [5], Theorem 2.1.4).

Given $A \geq 0$, the matrix

$$
B=s I-A \quad s>0,
$$

is called a Z-matrix. If in addition $s \geq \rho(A)$, then $B$ is called an M-matrix. In particular, an M-matrix $B$ in which $s>\rho(A)$ is nonsingular and such that $-B$ is asymptotically stable. If instead $s=\rho(A), B$ is a singular M-matrix. If in addition $A$ is irreducible, then $-B$ is also marginally stable.

In correspondence of eventually nonnegative matrices we have the following generalization of the class of M-matrices [17].

Definition 4 A matrix $B \in \mathbb{R}^{n \times n}$ is a $\mathbf{M}_{\vee}$-matrix if it is of the form $B=s I-A$ with $s \geq \rho(A)$ and $A \stackrel{\vee}{\geq}$.

The M-matrices form a proper subset of the $\mathbf{M}_{\vee}$-matrices. However, an $\mathrm{M}_{\vee}$-matrix need not be a Z-matrix, since it can have some positive off-diagonal entries.

Theorem 3 ([34], Theorem 3.4) Let $B \in \mathbb{R}^{n \times n}$ be an $M_{\vee}$ matrix, i.e., $B=s I-A$ with $A \geq 0$ and $s \geq \rho(A)$. Then

(i) $s-\rho(A) \in \operatorname{sp}(B)$;

(ii) $\operatorname{Re}\left[\lambda_{i}(B)\right] \geq 0 \forall \lambda_{i}(B) \in \operatorname{sp}(B)$;

(iii) $\operatorname{det}(B) \geq 0$ and $B$ is singular if and only if $s=\rho(A)$;

(iv) if $A$ nonnilpotent, then $\exists$ eigenvector $v_{r} \geq 0$ of $B$ and an eigenvector $v_{\ell} \geq 0$ of $B^{T}$ corresponding to $\lambda(B)=$ $s-\rho(A)$;

(v) if, in particular, $A \stackrel{\vee}{>} 0$ then in (iv) $v_{r}>0, v_{\ell}>0$;

(vi) if, in particular, $A \stackrel{\vee}{>} 0$ and $s>\rho(A)$ then in (iv) $v_{r}>0$, $v_{\ell}>0$, and in (ii) $\operatorname{Re}\left[\lambda_{i}(B)\right]>0 \forall \lambda_{i}(B) \in \operatorname{sp}(B)$.

In particular, if $B$ is an irreducible $\mathbf{M}_{\vee}$-matrix, then $-B$ is always at least marginally stable, and asymptotically stable if and only if $s>\rho(A)$.

Recall that a matrix $A \in \mathbb{R}^{n \times n}$ is said exponentially positive if $e^{A t}=\sum_{k=0}^{\infty} \frac{A^{k} t^{k}}{k !}>0 \forall t \geq 0$, and that $A$ is exponentially positive if and only if $A$ is essentially nonnegative, i.e., $a_{i j} \geq 0$ $\forall i \neq j[32]$.

Definition 5 A matrix $A \in \mathbb{R}^{n \times n}$ is said eventually exponentially positive if $\exists t_{o} \in[0, \infty)$ such that $e^{A t}>0 \forall t \geq t_{o}$.

We denote the smallest such $t_{o}$ the exponential index of $A$. The relationship between eventual positivity and eventual exponential positivity is provided by Theorem 3.3 of [32], recalled below for completeness.

Lemma 2 Given $A \in \mathbb{R}^{n \times n}, \exists d \geq 0$ such that $A+d I \stackrel{\vee}{>} 0$ if and only if $A$ is eventually exponentially positive.

\section{B. Invariant cones and eventually positive matrices}

A set $\mathcal{K} \subset \mathbb{R}^{n}$ is called a convex cone if $\alpha_{1} x+\alpha_{2} y \in \mathcal{K}$ $\forall x, y \in \mathcal{K}, \quad \alpha_{1}, \alpha_{2} \geq 0 . \mathcal{K}$ is called solid if the interior of $\mathcal{K}, \operatorname{int}(\mathcal{K})$, is nonempty and pointed if $\mathcal{K} \cap(-\mathcal{K})=\emptyset$ 
(where we have indicated $\emptyset=\{0\}$ ). A proper cone is a closed, pointed, solid cone. A cone is polyhedral if it can be expressed as the conical hull of a finite number of generating vectors $\omega_{1}, \ldots, \omega_{\mu} \in \mathbb{R}^{n}$ :

$$
\mathcal{K}=\operatorname{cone}(\Omega)=\left\{x=\sum_{i=1}^{\mu} \alpha_{i} \omega_{i}, \quad \alpha_{i} \geq 0\right\},
$$

where $\Omega=\left[\omega_{1} \ldots \omega_{\mu}\right] \in \mathbb{R}^{n \times \mu}, \alpha=\left[\alpha_{1} \ldots \alpha_{\mu}\right]^{T} \in \mathbb{R}_{+}^{\mu}$. Given $A \in \mathbb{R}^{n \times n}$, the cone $\mathcal{K}$ is said $A$-invariant if $A \mathcal{K} \subseteq \mathcal{K}$. For an $A$-invariant cone $\mathcal{K}, A$ is said $\mathcal{K}$-positive if $A(\mathcal{K} \backslash \emptyset) \subseteq$ $\operatorname{int}(\mathcal{K})$, i.e., $A$ maps any nonzero element of $\mathcal{K} \operatorname{into} \operatorname{int}(\mathcal{K})$. Notice that if $A$ is $\mathcal{K}$-positive then $A$ is $\mathcal{K}$-irreducible, i.e., it does not leave any of the faces of $\mathcal{K}$ invariant (except for $\emptyset$ and $\mathcal{K}$ itself). Theorem 1.3.16 of [5] says that $A$ that leaves $\mathcal{K}$ invariant is $\mathcal{K}$-irreducible if and only if $A$ has exactly one (up to scalar multiples) eigenvector in $\mathcal{K}$, and this vector is in $\operatorname{int}(\mathcal{K})$. Let $\mathcal{K}^{*}=\left\{y \in \mathbb{R}^{n}\right.$ s. t. $\left.y^{T} x \geq 0 \forall x \in \mathcal{K}\right\}$ be the dual cone of $\mathcal{K}$. Then $A$ is $\mathcal{K}$-positive if and only if $A^{T}$ is $\mathcal{K}^{*}$-positive [5].

The following theorem extends the Perron-Frobenius theorem to invariant cones (see e.g. Theorem 1.3.26 of [5], or Theorem 3.3 of [43]).

\section{Theorem 4 Given $A \in \mathbb{R}^{n \times n}$, the following are equivalent:}

(i) $\exists$ a proper A-invariant cone $\mathcal{K} \in \mathbb{R}^{n}$ for which $A$ is $\mathcal{K}$-positive;

(ii) $\rho(A)$ is a simple positive eigenvalue in $\mathrm{sp}(A)$, and for each $\lambda \in \operatorname{sp}(A), \lambda \neq \rho(A),|\lambda|<\rho(A)$.

Furthermore, the right eigenvector $v_{r}$ relative to $\rho(A)$ is $v_{r} \in$ $\operatorname{int}(\mathcal{K})$.

In the previous theorem, $\mathcal{K}$ can be taken to be polyhedral ([43], Theorem 3.3).

The following theorem links eventually positive matrices with invariant cones.

Theorem $5 A \stackrel{\vee}{>} 0$ if and only if $\exists$ a proper polyhedral $A$ invariant cone $\mathcal{K}$ such that $\mathcal{K} \subset \operatorname{int}\left(\mathbb{R}_{+}^{n}\right) \cup \emptyset, \mathcal{K}^{*} \subset \operatorname{int}\left(\mathbb{R}_{+}^{n}\right) \cup$ $\emptyset$, and $A$ is $\mathcal{K}$-positive.

Proof. One implication is straightforward: if $\exists \mathcal{K}$ such that $\mathcal{K} \subset \operatorname{int}\left(\mathbb{R}_{+}^{n}\right) \cup \emptyset$ and such that $A$ is $\mathcal{K}$-positive, then, from Theorem $4, \rho(A)$ is a simple positive eigenvalue strictly dominating all other eigenvalues. Positivity of $A$ on $\mathcal{K}$ implies that the corresponding eigenvector $v_{r} \in \operatorname{int}(\mathcal{K})$, hence $v_{r}>0$. Since $A$ is $\mathcal{K}$-positive if and only if $A^{T}$ is $\mathcal{K}^{*}$ positive, the condition $\mathcal{K}^{*} \subset \operatorname{int}\left(\mathbb{R}_{+}^{n}\right) \cup \emptyset$ implies that also for $v_{\ell}$, the left eigenvector of $A$ relative to $\rho(A), v_{\ell}>0$. Hence $A, A^{T} \in \mathcal{P} \mathcal{F}_{n}$ and Theorem 1 applies. As for the opposite implication, $A \stackrel{\vee}{>} 0$ implies $A \in \mathcal{P F}_{n}$ and the right eigenvector of $A$ relative to $\rho(A)$ is $v_{r}>0$. From Theorem 4, $\exists$ a proper cone $\mathcal{K}_{0}$ which is $A$-invariant and for which $A$ is $\mathcal{K}_{0}$-positive. Since $v_{r} \in \operatorname{int}\left(\mathcal{K}_{0}\right), \operatorname{int}\left(\mathcal{K}_{0}\right) \cap \operatorname{int}\left(\mathbb{R}_{+}^{n}\right) \neq \emptyset$, although in general $\mathcal{K}_{0} \not \subset \mathbb{R}_{+}^{n}$. Since $\mathcal{K}_{0}$ is polyhedral, it is (finitely) generated by the nonnegative combinations of certain $\mu_{0}$ vectors $\omega_{1}^{0}, \ldots, \omega_{\mu_{0}}^{0}$. Applying the linear operator $A$ to $\mathcal{K}_{0}$, then also $\mathcal{K}_{1}=A \mathcal{K}_{0}$ must be finitely generated by a number $\mu_{1} \leq \mu_{0}$ of vectors $\omega_{i}^{1}=A \omega_{i}^{0}, i=1, \ldots, \mu_{1}$. $A$-invariance implies $\mathcal{K}_{1}=A \mathcal{K}_{0} \subseteq \mathcal{K}_{0}$, and, iterating, $\mathcal{K}_{p+1}=A \mathcal{K}_{p}=A^{p+1} \mathcal{K}_{0} \subseteq A^{p} \mathcal{K}_{0}=\mathcal{K}_{p}$, i.e., each $\mathcal{K}_{p}$ is $A$-invariant. The sequence of invariant subcones

$$
\mathcal{K}_{0} \supseteq \mathcal{K}_{1} \supseteq \ldots \supseteq \mathcal{K}_{p} \supseteq \ldots
$$

terminates at the "core" of $\mathcal{K}_{0}: \mathcal{K}_{\infty}=\cap_{i=0}^{\infty} \mathcal{K}_{i} . A^{k}>0$ for all $k \geq k_{o}$, hence, from Theorem 4.1 of [35], $\mathcal{K}_{\infty}$ must be the single ray $v_{r}$. To show that for each $\mathcal{K}_{p} A$ is $\mathcal{K}_{p^{-}}$ positive, assume $A$ is $\mathcal{K}_{p-1}$-positive and $\mathcal{K}_{p} \not \subset \operatorname{int}\left(\mathcal{K}_{p-1}\right)$. This means that at least one of the $\mu_{p-1}$ generators of $\mathcal{K}_{p-1}$, $\omega_{i}^{p-1}=A^{p-1} \omega_{i}^{0}, i=1, \ldots, \mu_{p-1}$, is left invariant by $A$ : $\omega_{i}^{p}=A \omega_{i}^{p-1}$ for some $i$. This however implies that $\omega_{i}^{p-1}$ must be a generator of $\mathcal{K}_{q}$ for all $q \geq p-1$, because $A^{q} \omega_{i}^{p-1}=\omega_{i}^{p-1}$. Since $v_{r} \in \operatorname{int}\left(\mathcal{K}_{p-1}\right)$, it must also be $\omega_{i}^{p-1} \neq v_{r}$. But now we have a contradiction, as $\mathcal{K}_{\infty}$ cannot contain any other vector than $v_{r}$.A must therefore be $\mathcal{K}_{p^{-}}$ positive for each $p$ and hence the sequence (3) must be nested by strict inclusion. Since $\mathcal{K}_{\infty}$ is a single ray, the sequence (3) must be converging, with $v_{r}$ belonging to each $\mathcal{K}_{p}$. Hence there must exists an index $p_{o}$ for which $\mathcal{K}_{p} \subset \operatorname{int}\left(\mathbb{R}_{+}^{n}\right) \cup \emptyset$ for all $p \geq p_{o}$. An identical argument for $\mathcal{K}^{*}$ leads to $\mathcal{K}^{*} \subset \operatorname{int}\left(\mathbb{R}_{+}^{n}\right) \cup \emptyset$.

By construction, the cone $\mathcal{K}$ of Theorem 5 contains no other eigenvector of $A$ than $v_{r}$. It follows from the theorem that also $-\mathcal{K}$, for which $-\mathcal{K} \subset \operatorname{int}\left(\mathbb{R}_{-}^{n}\right) \cup \emptyset$, is an $A$-invariant cone, and $A$ is $(-\mathcal{K})$-positive. Similarly, $\mathcal{K}^{*}$ is such that $-\mathcal{K}^{*} \subset$ $\operatorname{int}\left(\mathbb{R}_{-}^{n}\right) \cup \emptyset$ and $A^{T}$ is $\left(-\mathcal{K}^{*}\right)$-positive.

The following corollary is a straightforward consequence of Theorem 5.

Corollary $1 A \stackrel{\vee}{>} 0$ but $A \nsupseteq 0$ implies that $\mathcal{K}$ of Theorem 5 cannot coincide with $\mathbb{R}_{+}^{n}$.

If instead of $A>0$ we have the weaker condition $A \in \mathcal{P} \mathcal{F}_{n}$, then Theorem 5 can be replaced by the following corollary, whose proof is analogous to that of Theorem 5 .

Corollary $2 A \in \mathcal{P F}_{n}$ if and only if $\exists$ a proper polyhedral $A$-invariant cone $\mathcal{K}$ such that $\mathcal{K} \subset \operatorname{int}\left(\mathbb{R}_{+}^{n}\right) \cup \emptyset$ and $A$ is $\mathcal{K}$-positive.

\section{UNANIMITY OF OPINION}

Consider a strongly connected signed digraph $\Gamma(A)$ whose adjacency matrix $A=\left(a_{i j}\right) \in \mathbb{R}^{n \times n}$ is such that $a_{i i}=0$. We assume that a distributed process of opinion forming takes place on $\Gamma(A)$ through the associated linear dynamical system

$$
\dot{x_{i}}=-\sigma_{i} x_{i}+\sum_{j \in \operatorname{adj}(i)} a_{i j} x_{j}, \quad i=1, \ldots, n,
$$

where $\sigma_{i}>0, i=1, \ldots n$, are called the degradation rates of the interconnected system and represent forgetting factors for the opinions. Denoting $x=\left[x_{1}, \ldots, x_{n}\right]^{T} \in \mathbb{R}^{n}$ and $\Sigma=$ $\operatorname{diag}\left(\sigma_{1}, \ldots, \sigma_{n}\right)$, the system (4) is then written in matrix form as

$$
\dot{x}=E x, \quad E=A-\Sigma \in \mathbb{R}^{n \times n} .
$$


When $a_{i j} \geq 0$ then the system (5) is said a cooperative (or positive) system. We are here interested in the more general case of $A$ having some negative entries.

\section{A. Predicting unanimous opinions via eventually positive ad- jacency matrices}

For eventually positive matrices the strong Perron-Frobenius property can be used to predict the formation of unanimous opinions. The following theorem highlights the role of the spectral radius in this context.

Theorem 6 Consider the system (5), with $A \stackrel{\vee}{>} 0$.

(i) If $\sigma_{i} \geq \rho(A), \forall i=1, \ldots, n$, and $\exists$ at least one $\sigma_{i}$ such that $\sigma_{i}>\rho(A)$, then $x^{*}=\lim _{t \rightarrow \infty} x(t)=0$;

(ii) If $\sigma_{i}=\rho(A), \forall i=1, \ldots, n$, then $x^{*}=\frac{1}{v_{\ell}^{T} v_{r}} v_{\ell}^{T} x_{o} v_{r}$, where $v_{\ell}$ and $v_{r}$ are respectively the left and right eigenvector of $A$ associated to $\rho(A)$, and $x_{o} \in \mathbb{R}^{n}$ the initial condition;

(iii) If $\sigma_{i} \leq \rho(A), \forall i=1, \ldots, n$, and $\exists$ at least one $\sigma_{i}$ such that $\sigma_{i}<\rho(A)$, then $x^{*}= \pm \infty$.

Proof. See Appendix.

\section{B. Holdability and unanimity of an opinion}

Recall (see e.g. [7]) that a set $\mathcal{Y} \subset \mathbb{R}^{n}$ is said (positively) invariant for a dynamical system

$$
\dot{x}=f(x)
$$

if for any initial condition $x_{o}=x(0) \in \mathcal{Y}$ its trajectories $x(t) \in \mathcal{Y} \forall t \geq 0$. A set $\mathcal{Y} \subset \mathbb{R}^{n}$ is said instead attractive for (6) if $\forall x_{o} \in \mathbb{R}^{n} \lim _{t \rightarrow \infty} \operatorname{dist}(x(t), \mathcal{Y})=0$ where $\operatorname{dist}(x(t), \mathcal{Y})=\inf _{y \in \mathcal{Y}}\|x(t)-y\|$, with $\|\cdot\|$ any norm in $\mathbb{R}^{n}$. We will say further that an attractive set $\mathcal{Y}$ is holdable for (6) if for any $x_{o} \in \mathbb{R}^{n} \exists t_{o}=t_{o}\left(x_{o}\right) \geq 0$ such that $x(t) \in \mathcal{Y}$ for $t \geq t_{o}$. Notice that set attractivity and set holdability are closely related concepts. However, since $\mathcal{Y}$ can be unbounded, holdability does not imply convergence to an equilibrium point nor to a bounded trajectory, hence we prefer to maintain a distinct terminology. Notice further that as an invariant set need not be attractive, $\mathcal{Y}$ invariant does not imply $\mathcal{Y}$ holdable. As for the opposite implication, $\mathcal{Y}$ holdable does not imply $\mathcal{Y}$ invariant: $x$ can enter $\mathcal{Y}$, exit it and reenter definitively at later times.

Let $\mathcal{S}$ be the set of partial orthant orders in $\mathbb{R}^{n}: \mathcal{S}=$ $\left\{s=\left[\begin{array}{lll}s_{1} & \ldots & s_{n}\end{array}\right]^{T}, s_{i}= \pm 1\right\}$. Given $s \in \mathcal{S}$, denote $S=\operatorname{diag}(s)$ and let $\mathbb{R}_{s}^{n}$ be the corresponding orthant: $\mathbb{R}_{s}^{n}=\left\{x \in \mathbb{R}^{n}\right.$ s. t. $\left.S x \geq 0\right\}$. The wedge obtained joining two "opposite" orthants is denoted $\mathbb{R}_{\{-s, s\}}^{n}=\mathbb{R}_{s}^{n} \cup \mathbb{R}_{-s}^{n}$. In particular we indicate $\mathbb{R}_{\{-,+\}}^{n}=\left\{x \in \mathbb{R}^{n}\right.$ s. t. $x \leq 0$ or $x \geq$ $0\}$. In this paper we are interested in systems that holds to the orthant pair $\mathbb{R}_{\{-s, s\}}^{n}$, i.e., such that for each $x_{o} \in \mathbb{R}^{n} \exists$ $t_{o}=t_{o}\left(x_{o}\right) \geq 0$ such that $x(t) \in \mathbb{R}_{\{-s, s\}}^{n} \forall t \geq t_{o}$. When $s=\mathbf{1}=[1 \ldots 1]^{T}$ we will also say that the system achieves an unanimous opinion. In this case a system with the holdability property is a generalization of a positive system, in which $\mathbb{R}_{+}^{n}$ (or $\mathbb{R}_{-}^{n}$ ) is not invariant for the system but still all trajectories are attracted to it, after a transient excursion.
In the following we will link the notion of unanimity to the existence of an invariant cone that holds to the positive orthant. For that, let us recall the basic necessary and sufficient condition for the existence of an invariant cone for linear systems [6], [7], [12], [40], [41].

Proposition 1 ([12], Proposition 1) Consider the system (5). The cone $\mathcal{K}=\operatorname{cone}(\Omega)$ is invariant for (5) if and only if $\exists$ an essentially nonnegative matrix $H \in \mathbb{R}^{\mu \times \mu}$ such that

$$
E \Omega=\Omega H \text {. }
$$

The rationale of the proof of Proposition 1 is that by recursively multiplying an expression like (7) to the right by $E$ one gets

$$
E^{k} \Omega=E\left(E^{k-1} \Omega\right)=E \Omega H^{k-1}=\Omega H^{k}
$$

and hence, summing up,

$$
e^{E t} \Omega=\Omega e^{H t}
$$

When the invariant cone satisfies Theorem 5 , then it can be used to characterize unanimity. Notice that even if $A \stackrel{\vee}{>}$, a system like (5) with $E=A-\Sigma$ need not necessarily converge to an unanimous opinion when $\Sigma$ is not proportional to the identity, because $A \stackrel{\vee}{>} 0 \not E \stackrel{\vee}{>} 0$. When this happens, however, the system (5) holds to the orthant pair $\mathbb{R}_{\{-,+\}}^{n}$, see also [32] for related material. A more general sufficient condition is provided by the following theorem.

Theorem 7 Consider the system (5). If $\exists d \in \mathbb{R}$ such that $A+D \stackrel{\vee}{>} 0$, where $D=d I-\Sigma$, then the system (5) holds to the orthant pair $\mathbb{R}_{\{-,+\}}^{n}$.

Proof. Denote $B=A+D \stackrel{\vee}{>} 0$ of spectral radius $\rho(B)$ and of left and right eigenvectors $v_{\ell}>0$ and $v_{r}>0$. Since $\Sigma=$ $d I-D$

$$
E=A-\Sigma=B-D-d I+D=B-d I,
$$

which implies that $E$ must have $\lambda_{i}(B)-d$ as eigenvalues. In particular, then, $\rho(B)-d$ (of multiplicity 1) must be the eigenvalue of $E$ of largest real part and $v_{\ell}>0, v_{r}>0$ its left and right eigenvectors. Since $B$ and $d I$ commute, we can write

$$
e^{E t}=e^{B t} e^{-d t},
$$

from which it follows that the system (5) converges to the Perron-Frobenius eigenspace, $x^{*} \in \operatorname{span}\left(v_{r}\right)$, and in particular $x^{*} \in \operatorname{int}\left(\mathbb{R}_{+}^{n}\right) \cup \emptyset$ if $v_{\ell}^{T} x_{o}>0$ and $x^{*} \in \operatorname{int}\left(\mathbb{R}_{-}^{n}\right) \cup \emptyset$ if $v_{\ell}^{T} x_{o}<0$, with $x_{o} \in \mathbb{R}^{n}$ the initial condition. Hence $\mathbb{R}_{\{-,+\}}^{n}$ is an attractive set. From (9), the nonnegative scalar factor $e^{-d t}$ does not change this conclusion (it only alters the value of $x^{*}$ in $\operatorname{span}\left(v_{r}\right)$ ). Let us consider the case $v_{\ell}^{T} x_{o}>0$. Since $B \stackrel{\vee}{>} 0$, from Theorem $5 \exists$ a proper $B$-invariant polyhedral cone $\mathcal{K}$ s. t. $\mathcal{K} \subset \operatorname{int}\left(\mathbb{R}_{+}^{n}\right) \cup \emptyset$ for which $B$ is $\mathcal{K}$-positive and hence $v_{r} \in \operatorname{int}(\mathcal{K})$. We will now show that the system (5) is invariant with respect to this cone. If $\mathcal{K}=\operatorname{cone}(\Omega)$, with $\Omega \in \mathbb{R}^{n \times \mu}$ full row rank, by construction, $B$-invariance of $\mathcal{K}$ implies $\exists H_{B} \in \mathbb{R}_{+}^{\mu \times \mu}$ such that $B \Omega=\Omega H_{B}$. Therefore 
$(B-d I) \Omega=\Omega\left(H_{B}-d I\right)$, or (7), with $H=H_{B}-d I$ an essentially nonnegative matrix. From Proposition 1 , the system (5) is invariant on $\mathcal{K}$. Since $x^{*} \in \operatorname{int}(\mathcal{K}) \cup \emptyset$ and also $E$ is $\mathcal{K}$-positive, by continuity, each trajectory of (5) belongs to $\mathcal{K}$ for times sufficiently long. In particular, for each $x_{o} \in \mathbb{R}^{n} \exists$ $t_{o}=t_{o}\left(x_{o}\right)$ such that $x(t) \in \mathcal{K} \forall t \geq t_{o}$. Hence (5) holds to $\mathbb{R}_{+}^{n}$. For the case $v_{\ell}^{T} x_{o}<0$, the proof is analogous if one considers the negated cone $-\mathcal{K}$. From $-\mathcal{K} \subset \operatorname{int}\left(\mathbb{R}_{-}^{n}\right) \cup \emptyset$, in this case we have that (5) holds to $\mathbb{R}_{-}^{n}$.

The sufficient condition of Theorem 7 can be readily weakened to a "one-sided" Perron-Frobenius property, although at the practical cost of less efficient numerical tests (it is no longer enough to compute powers of a matrix).

Corollary 3 If $\exists d \in \mathbb{R}$ such that $A+D \in \mathcal{P} \mathcal{F}_{n}$, where $D=d I-\Sigma$, then the system (5) holds to the orthant pair $\mathbb{R}_{\{-,+\}}^{n}$.

Proof. If $B=A+D \in \mathcal{P} \mathcal{F}_{n}$ then the right eigenvector $v_{r}$ relative to $\rho(B)$ is $v_{r}>0$ and, from Corollary 2, $\exists$ a proper cone $\mathcal{K}$ contained in $\operatorname{int}\left(\mathbb{R}_{+}^{n}\right) \cup \emptyset$ such that $B$ is $\mathcal{K}$-positive and $v_{r} \in \operatorname{int}(\mathcal{K})$. The proof that the system (5) is invariant for this cone is identical to that of Theorem 7 and so is the conclusion.

The sufficient condition of Corollary 3 is probably very close to necessity, although it is not clear how to prove it.

Example 2 Consider the signed network whose adjacency matrix is

$$
A=\left[\begin{array}{ccc}
0 & 1.7877 & -0.6743 \\
-0.7678 & 0 & 0.7354 \\
0.5878 & 0 & 0
\end{array}\right]
$$

$A$ is not eventually nonnegative; however, choosing $D=$ $\operatorname{diag}(0.2688,1.002,1.3272)$, the matrix $B=A+D \stackrel{\vee}{>} 0$. As in the proof of Theorem 5 , it is possible to construct a sequence of nested $B$-invariant polyhedral cones $\mathcal{K}_{p}=B \mathcal{K}_{p-1}$ for which $B$ is $\mathcal{K}_{p}$-positive, starting from a $\mathcal{K}_{0}$ constructed e.g. following the procedure described in [43]. In this case $B^{p}>0$ for $p \geq 19$ and $\mathcal{K}_{p} \subset \mathbb{R}_{+}^{3}$ only for $p \geq 19$. The sequence of $\mathcal{K}_{p}$ is shown in Fig. 1. The matrix $B$ has $\operatorname{sp}(B)=\{1.5817,0.5082 \pm 1.3635 i\}$ and

$$
\begin{aligned}
& v_{\ell}=\left[\begin{array}{lll}
0.1418 & 0.4373 & 0.8880
\end{array}\right]^{T} \\
& v_{r}=\left[\begin{array}{lll}
0.3350 & 0.5378 & 0.7737
\end{array}\right]^{T} .
\end{aligned}
$$

Denoting $\Sigma=d I-D$, when $d=\rho(B)$ we obtain that $E$ is a singular negated $\mathrm{M}_{\vee}$-matrix and that the system (5) converges to the ray determined by $v_{r}$ (visible in black in Fig. 1 inside $\mathcal{K}_{p}$ ). A trajectory of the system is also shown in Fig. 1 (blue curve). Notice that $x(0)>0 \not x(t)>0 \forall t \geq 0$, i.e., $\mathbb{R}_{+}^{3}$ is not invariant, as expected. However, $x(t) \in \mathcal{K}_{p}$ for $t$ large enough, hence the system is $\mathbb{R}_{\{-,+\}}^{3}$ holdable.

Example 3 The network considered in this example consists of $n=100$ agents connected through $m=1000$ randomly chosen edges, of which 162 are negative and 838 positive. In

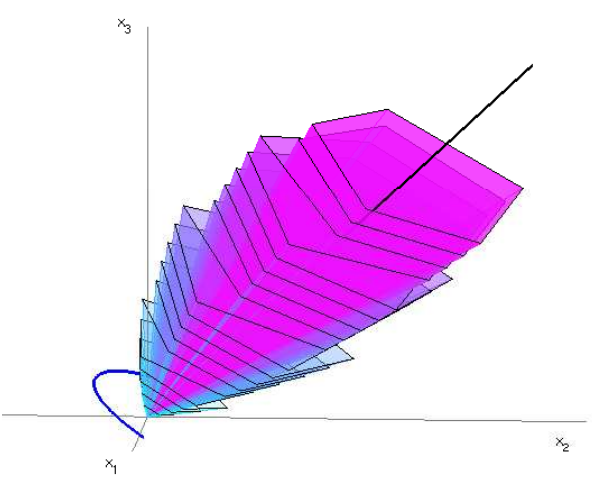

(a)

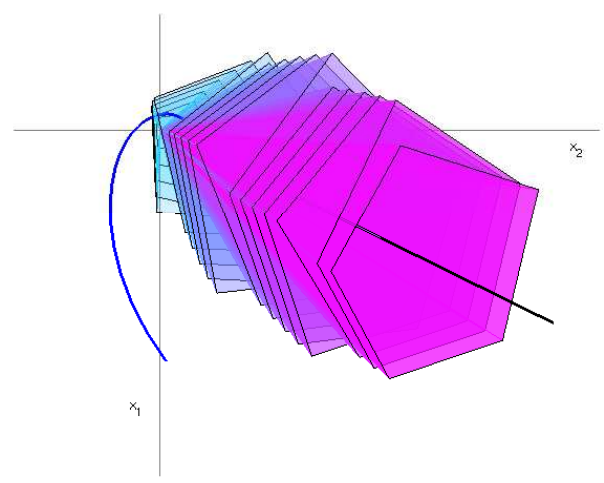

(b)

Fig. 1. Example 2. (a): 3D view. (b): top view of the same figure.

spite of the large number of negative entries, $A^{k}>0$ already for $k \geq k_{o}=10$. The three cases mentioned in Theorem 6 (in correspondence of three different choices of $\Sigma$ ) are illustrated in Fig. 2 for two different values of $x_{o}$. In both panels (b) and (c) the top plot corresponds to a nonsingular negated $\mathrm{M}_{\vee}$ matrix $E$ and the middle plot to a singular negated $\mathbf{M}_{\vee}$-matrix $E$, while in the bottom plot $E$ is not a negated $\mathbf{M}_{\vee}$ matrix. In this example $\mathbb{R}_{\{-,+\}}^{100}$ is holdable even with $d=0(E \stackrel{\vee}{>} 0$ in all 3 cases). In the top plots $x^{*}=0$ is approached from $\mathbb{R}_{+}^{100}$ (panel (b)) or from $\mathbb{R}_{-}^{100}$ (panel (c)). In the middle plots, all components of $x^{*}$ have the same sign. Similarly, in the bottom plots they diverge all to $+\infty$ or all to $-\infty$. In all three cases $x^{*} \in \operatorname{span}\left(v_{r}\right)$.

\section{Cooperation need not preserve unanimity}

Unlike for nonnegative matrices, the set $\mathcal{P} \mathcal{F}_{n}$ is not convex, although by continuity each $A \in \mathcal{P} \mathcal{F}_{n}$ is at the center of a pointed cone of matrices in $\mathcal{P} \mathcal{F}_{n}$ [26], cone whose width is difficult to quantify. As a consequence, a convex combination of eventually positive matrices need not be eventually positive as the following example shows.

Example 4 Consider the convex combinations $F=\alpha B+$ 


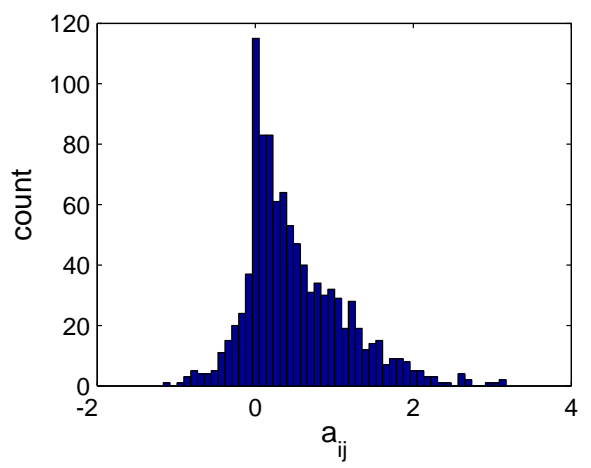

(a)
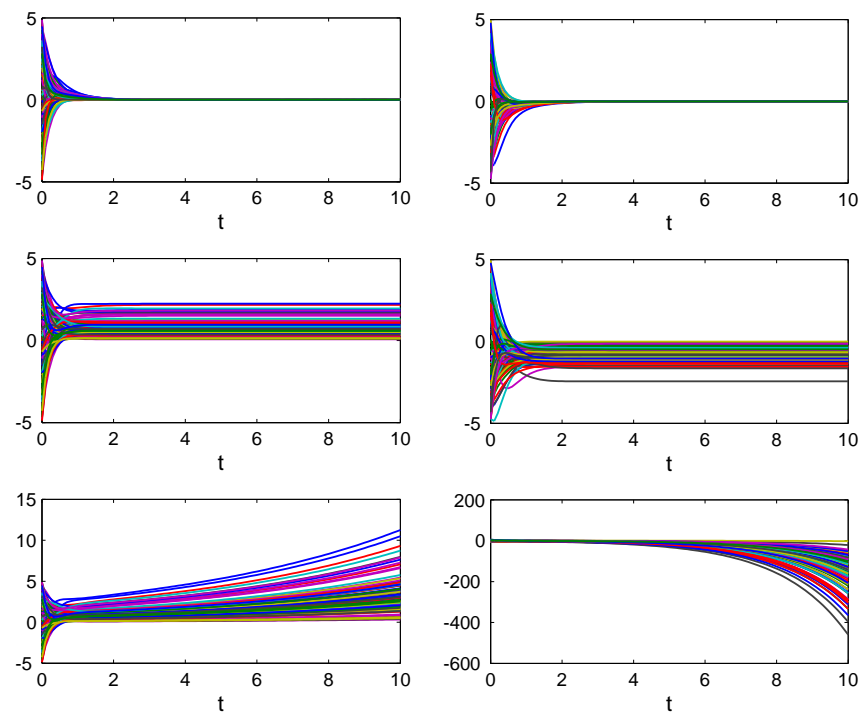

(b)

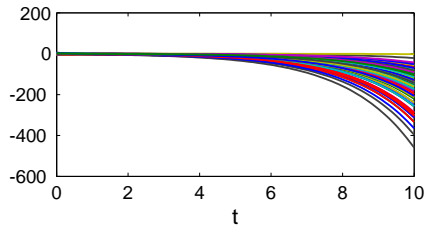

(c)

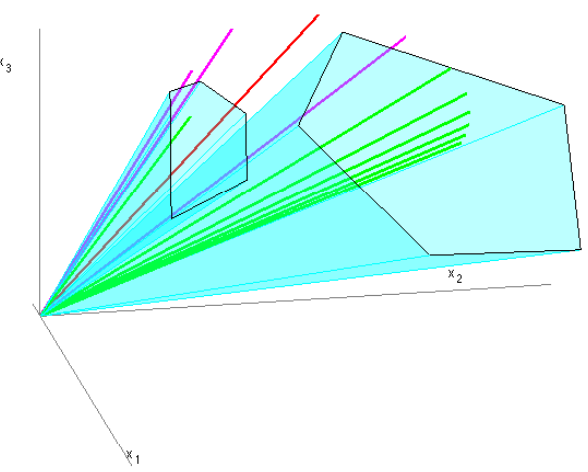

(a)

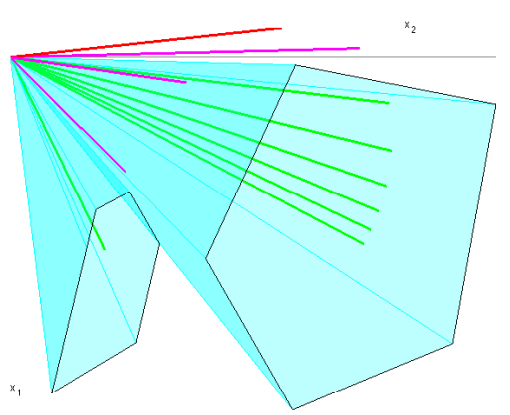

(b)

Fig. 2. Example 3. In (a) the distribution of the nonzero edges of $A$ is shown. In (b) and (c), from top to bottom, the asymptotically stable case, the marginally stable case and the unstable case are shown for two different initial conditions.

$(1-\alpha) B_{1}$, where $0 \leq \alpha \leq 1, B \stackrel{\vee}{>} 0$ is as in Example 2 and

$$
B_{1}=\left[\begin{array}{ccc}
0.325 & -1.169 & 0.411 \\
0.242 & 0.357 & 0 \\
0 & 0.001 & 0.938
\end{array}\right] \stackrel{\vee}{>} 0 .
$$

As can be seen in Fig. 3, although there exists two cones for $B$ and for $B_{1}$ both sitting inside $\operatorname{int}\left(\mathbb{R}_{+}^{3}\right)$ and $\rho(F)$ is a simple positive strictly dominating eigenvalue for all $\alpha \in$ $[0,1]$, in correspondence of $\rho(F), v_{r}$ and/or $v_{\ell}$ do not remain nonnegative as $\alpha$ changes from 0 to 1 . The color-code of Fig. 3 corresponds to $F>0$ (green), $F \notin \mathcal{P} \mathcal{F}_{3}$ but $F^{T} \notin \mathcal{P} \mathcal{F}_{3}$ or viceversa (magenta), and $F, F^{T} \notin \mathcal{P} \mathcal{F}_{3}$ (red).

In addition, we also have that even convex combinations of eventually positive matrices and nonnegative matrices are not necessarily eventually positive.

Example 5 Let us consider again $B \stackrel{\vee}{>} 0$ of Example 2. If we

add to it

$$
B_{2}=\left[\begin{array}{ccc}
0 & 0 & 0.8 \\
0 & 0 & 0 \\
0 & 2 & 0
\end{array}\right]
$$

then $B+B_{2}$ is not eventually positive, although $\rho\left(B+B_{2}\right)$ is a simple strictly dominating positive eigenvalue of $v_{r}>0$. In this case $B+B_{2} \in \mathcal{P} \mathcal{F}_{3}$, but $\left(B+B_{2}\right)^{T} \notin \mathcal{P} \mathcal{F}_{3}$, i.e., $v_{\ell}$ is not $>0$. If instead of $B_{2}$ we add

$$
B_{3}=\left[\begin{array}{ccc}
0 & 0 & 2.3 \\
0 & 0 & 0 \\
0 & 2.5 & 0
\end{array}\right]
$$

then neither $B+B_{3}$ nor $\left(B+B_{3}\right)^{T}$ is in $\mathcal{P F}_{3}$.

\section{Achieving unanimity in presence of stubborn agents}

According to [22], [1], [21], a stubborn agent is a node whose opinion is not influenced by those of the other agents but that can exercise on them an influence as any other node. Denoting $z$ the opinion of the stubborn agents and $z_{o}$ their initial condition, then "total stubbornness" corresponds to $z(t)=$ const $=z_{o} \forall t \geq 0$. The continuous-time model of a network with $n$ "ordinary" (i.e., influenceable) agents and 
$r$ totally stubborn agents is therefore

$$
\begin{aligned}
\dot{x} & =E x+C z \\
\dot{z} & =0
\end{aligned}
$$

where $E=A-\Sigma$ is as in (5) and $C \in \mathbb{R}^{n \times r}$ is the matrix describing how the stubborn agents influence the susceptible ones. The case studied in the literature [22], [21] corresponds to $A \geq 0$ and $C \geq 0$. For it the problem of interest here, achieving unanimity, is completely trivial, at least as long as all $z_{i}$ have the same sign. When instead $A$ and $C$ are signed matrices, understanding to what extent and how $z$ can influence unanimity is far from trivial.

In the following this problem is recast as a cone invariance problem in presence of a persistent disturbance, which can be solved following the approach proposed by [29]. Denote $\mathcal{K}_{z}=\operatorname{cone}(\Psi)$ a closed convex polyhedral cone containing the opinions of the stubborn agents. Analogously to (2), $\Psi \in$ $\mathbb{R}^{r \times \eta}$ is a full row rank matrix whose columns represent the generating vectors of the cone. A set $\mathcal{Y}$ is said a (positively) $\mathcal{K}_{z}$-invariant set for the system (10) if $\forall x_{o} \in \mathcal{Y}$ its trajectories $x(t) \in \mathcal{Y}, \forall z \in \mathcal{K}_{z}$ and $\forall t \geq 0$.

Proposition 2 ([29], Proposition 1) Consider the system (10)(11), where $z_{o} \in \mathcal{K}_{z}=$ cone $(\Psi)$. The E-invariant cone $\mathcal{K}=$ cone $(\Omega)$ is $\mathcal{K}_{z}$-invariant for the system (10) if and only if $\exists$ an essentially nonnegative matrix $H \in \mathbb{R}^{\mu \times \mu}$ such that

$$
E \Omega=\Omega H
$$

and $\exists$ a nonnegative matrix $K \in \mathbb{R}^{\mu \times \eta}$ such that

$$
C \Psi=\Omega K \text {. }
$$

The proof of this Proposition is clearly inspired by Proposition 1 of [29], which however uses the dual (face) description of a polytope. It is reported here only because several steps are needed in the proof of the Theorem that follows.

Proof. Assume $x(t) \in \mathcal{K} \forall z_{o} \in \mathcal{K}_{z}$ and $\forall t \geq 0$. Writing $y=\left[\begin{array}{l}x \\ z\end{array}\right]$, then (10)-(11) become

$$
\dot{y}=\left[\begin{array}{cc}
E & C \\
0 & 0
\end{array}\right] y=F y .
$$

Let us consider the augmented cone for $y$ :

$$
\mathcal{K}_{y}=\left\{y=\left[\begin{array}{ll}
\Omega & 0 \\
0 & \Psi
\end{array}\right]\left[\begin{array}{l}
\alpha \\
\beta
\end{array}\right], \alpha \geq 0, \beta \geq 0\right\} .
$$

Since $x \in \mathcal{K}$ and $z=z_{o} \in \mathcal{K}_{z}$, by construction $\mathcal{K}_{y}$ is an $F$ invariant cone for (10)-(11), hence from Proposition $1 \exists M \in$ $\mathbb{R}^{(\mu+\eta) \times(\mu+\eta)}$ essentially nonnegative such that

$$
\left[\begin{array}{cc}
E & C \\
0 & 0
\end{array}\right]\left[\begin{array}{cc}
\Omega & 0 \\
0 & \Psi
\end{array}\right]=\left[\begin{array}{cc}
\Omega & 0 \\
0 & \Psi
\end{array}\right]\left[\begin{array}{ll}
M_{11} & M_{12} \\
M_{21} & M_{22}
\end{array}\right],
$$

where $M_{11} \in \mathbb{R}^{\mu \times \mu}, M_{22} \in \mathbb{R}^{\eta \times \eta}$ are essentially nonnegative and $M_{12} \in \mathbb{R}^{\mu \times \eta}, M_{21} \in \mathbb{R}^{\eta \times \mu}$ are nonnegative. Multiplying and comparing:

$$
\begin{aligned}
E \Omega & =\Omega M_{11} \\
C \Psi & =\Omega M_{12} \\
M_{21} & =0 \\
M_{22} & =0,
\end{aligned}
$$

and (12)-(13) follow if we call $H=M_{11}$ and $K=M_{12}$. As for the opposite implication, assume (12)-(13) hold. From Proposition 1, (12) implies that $\exists$ an $E$-invariant cone $\mathcal{K}=$ cone $(\Omega)$ and hence that (8) holds. Expanding the solution of (10),

$$
x(t)=e^{E t} x_{o}+\int_{0}^{t} e^{E(t-\tau)} C z_{o} d \tau,
$$

where any $x_{o}$ can be written as $x_{o}=\Omega \alpha$ for some $\alpha \geq 0$ and, since by construction $z_{o} \in \mathcal{K}_{z}$, also $z_{o}=\Psi \beta$ for some $\beta \geq 0$. Hence

$$
\begin{aligned}
x(t) & =e^{E t} \Omega \alpha+\int_{0}^{t} e^{E(t-\tau)} C \Psi \beta d \tau \\
& =\Omega e^{E t} \alpha+\int_{0}^{t} e^{E(t-\tau)} \Omega K \beta d \tau \\
& =\Omega e^{E t} \alpha+\int_{0}^{t} \Omega e^{H(t-\tau)} K \beta d \tau,
\end{aligned}
$$

where we have applied (8) and (13). Furthermore, since $H$ is essentially nonnegative and $K \geq 0, \alpha^{\prime}=e^{E t} \alpha \geq 0$ and $\beta^{\prime}=e^{H(t-\tau)} K \beta \geq 0$, meaning that

$$
x(t)=\Omega \alpha^{\prime}+\Omega \int_{0}^{t} \beta^{\prime}(\tau) d \tau \in \mathcal{K} \quad \forall t \geq 0
$$

which concludes the proof.

We can now combine invariance and unanimity in presence of stubborn agents.

Theorem 8 Consider the system (10)-(11) with $E=A-\Sigma$, $z_{o} \in \mathcal{K}_{z}$, and assume $\exists d \in \mathbb{R}$ such that $B=A+D \stackrel{\vee}{>} 0$, where $D=d I-\Sigma$. Consider the corresponding $B$-invariant polyhedral cone $\mathcal{K}$ for which $B$ is $\mathcal{K}$-positive. If $\exists k_{1} \in \mathbb{N}$ s.t. $B^{k} C \geq 0 \forall k>k_{1}$ then $\mathcal{K}$ is $\mathcal{K}_{z}$-invariant and the system (10) holds to the orthant pair $\mathbb{R}_{\{+,-\}} \forall z_{o} \in \mathcal{K}_{z}$.

Proof. Rewriting (10)-(11) as in (14), and calling

$$
F_{B}=\left[\begin{array}{cc}
B & C \\
0 & 0
\end{array}\right]
$$

it can be observed that $B \stackrel{\vee}{>} 0$ and $B^{k} C \geq 0 \forall k>k_{1}$ imply that $\exists k_{2} \geq \max \left(k_{o}, k_{1}\right)$ s.t.

$$
F_{B}^{k}=\left[\begin{array}{cc}
B^{k} & B^{k-1} C \\
0 & 0
\end{array}\right] \geq 0 \quad \forall k \geq k_{2},
$$

i.e. $F_{B}$ is eventually nonnegative. Since $B$ is nonnilpotent, and $F_{B}$ is block triangular, Theorem 2 applies, meaning that in correspondence of $\rho\left(F_{B}\right)$ the right eigenvector has to be nonnegative. Notice further that for any nonzero eigenvalue of $F_{B}, \lambda\left(F_{B}\right)$, the corresponding eigenvector $v=\left[\begin{array}{l}v_{x} \\ v_{z}\end{array}\right]$ must 
obey

$$
\begin{aligned}
B v_{x}+C v_{z} & =\lambda\left(F_{B}\right) v_{x} \\
0 & =\lambda\left(F_{B}\right) v_{z}
\end{aligned}
$$

or $v_{z}=0$ and $B v_{x}=\lambda\left(F_{B}\right) v_{x}$, meaning that only the eigenvalues of $B$ matter and that the corresponding eigenvectors are trivially extended to $F_{B}$ by adding zero components. Since $B \stackrel{\vee}{>} 0$, from Theorem $1 \rho(B)$ is a simple strictly dominating positive eigenvalue of eigenvector $v_{x}>0$. Hence, from Theorem $4 \exists$ a $B$-invariant cone $\mathcal{K}$ for which $B$ is $\mathcal{K}$ positive and $v_{x} \in \operatorname{int}(\mathcal{K})$. Furthermore, since $\rho\left(F_{B}\right)=\rho(B)$ is also a simple strictly dominating positive eigenvalue for $F_{B}$, then $\exists$ a cone $\mathcal{K}_{y}=$ cone $\left(\begin{array}{cc}\Omega & 0 \\ 0 & \Psi\end{array}\right)$ which is $F_{B}$-invariant, although $F_{B}$ is not $\mathcal{K}_{y}$-positive for it because of the triviality of its last $r$ rows. But then Proposition 1 holds for $F_{B}$ and, given the essential nonnegativity of the $M_{11}$ diagonal block, also an analogue of (15) holds, in which we have replaced $E$ with $B=E+d I$. Proposition 2 is therefore valid and the $\mathcal{K}_{z}$-invariance of $\mathcal{K}$ follows. Finally, from Theorem $5, \mathcal{K}$ can always be chosen so that $\mathcal{K} \subset \operatorname{int}\left(\mathbb{R}_{+}^{n}\right) \cup \emptyset$, hence holdability also follows.

Remark 1 Just like $B \stackrel{\vee}{>} 0 \not E=B-d I \stackrel{\vee}{>} 0$, so, in Theorem $8, B^{k} C \geq 0 \not E^{k} C \geq 0$. As in the previous cases, the role of $d$ is to change the asymptotic value $x^{*}$ along the eigenspace $\operatorname{span}\left(v_{x}\right)$ to which the system converges.

Example 6 Consider $A$ of Example 2, and $\Sigma=\operatorname{diag}(1.63,0.9,0.57)$, so that $E$ is asymptotically stable. If $d=1.9$ then $B \stackrel{\vee}{>} 0$, hence $\exists$ an invariant cone $\mathcal{K}$ that (perhaps after some iterations) lies inside $\mathbb{R}_{\{+,-\}}^{3}$. Assume $\exists$ a single stubborn agent with coupling matrix given by

$$
C_{1}=\left[\begin{array}{c}
0 \\
-1 \\
2.6
\end{array}\right]
$$

and that $\mathcal{K}_{z}=$ cone $(1)$, i.e., $z_{o}>0$. In this example, $z$ is influencing negatively $x_{2}$ and positively $x_{3}$, which makes it difficult to assess a priori the sign of the evolution of $x$, given the persistent nature of the excitation induced by $z$ (without it the system would instead converge to the origin). However, Theorem 8 holds, hence $\mathcal{K}$ is $\mathcal{K}_{z}$-invariant. A simulation for this case is shown in Fig. 4 (a). If we replace $C_{1}$ with

$$
C_{2}=\left[\begin{array}{c}
-9 \\
1.5 \\
2
\end{array}\right]
$$

then Theorem 8 no longer holds and in fact unanimity is lost, see Fig. 4 (b). As a matter of fact, since $E$ is asymptotically stable, the asymptotic solution of (10) is simply

$$
x^{*}=-E^{-1} C_{i} z_{o},
$$

which indeed confirms the values reported in the two cases of Fig. 4.

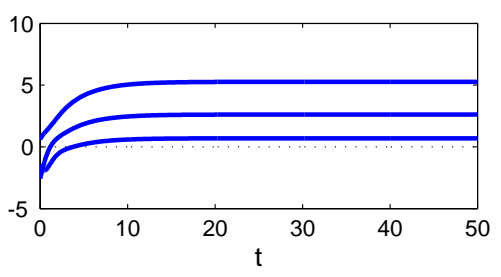

(a)

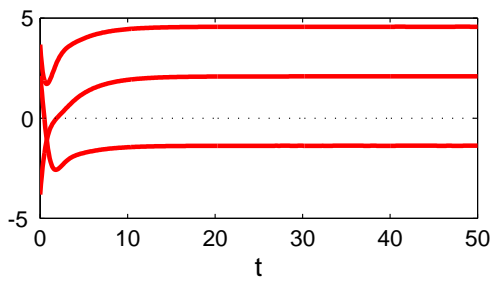

(b)

Fig. 4. Example 6. (a): $C_{1}$ coupling: unanimity is preserved in spite of the stubborn agent. (b): $C_{2}$ coupling: unanimity is not preserved in presence of the stubborn agent.

\section{CONTROL PROBLEMS FOR UNANIMOUS OPINIONS}

Let us consider a system of integrators on the signed graph $\Gamma(A)$

$$
\dot{x}_{i}=u_{i}, \quad i=1, \ldots, n .
$$

As before, we assume $A \in \mathbb{R}^{n \times n}$ is such that $a_{i i}=0$, $i=1, \ldots, n$. Our task in this section is to design feedback laws based on the state of the node and of its first neighbors, $u_{i}=g_{i}\left(x_{i}, x_{j}, j \in \operatorname{adj}(i)\right)$, in order to achieve an unanimous opinion. The system (16) with such a feedback law is distributed with respect to the topology of $\Gamma(A)$. Unlike in Theorem 6 , only nonzero, noninfinite steady states are normally considered interesting control objectives. The following is a direct consequence of Theorem 7 .

Proposition 3 Consider the system (16) on the signed graph $\Gamma(A)$. If $\exists D=\operatorname{diag}\left(d_{1}, \ldots, d_{n}\right)$ such that $A+D \stackrel{\vee}{>} 0$ then for any $d \in \mathbb{R}$ the system on $\Gamma(A)$ with the feedback

$$
u_{i}=\left(d_{i}-d\right) x_{i}+\sum_{j \in \operatorname{adj}(i)} a_{i j} x_{j}, \quad i=1, \ldots, n,
$$

achieves an unanimous opinion. In particular, if $d=\rho(A+D)$ then

$$
x^{*}=\lim _{t \rightarrow \infty} x(t)=\frac{v_{\ell}^{T} x_{o} v_{r}}{v_{\ell}^{T} v_{r}},
$$

where $v_{\ell}$ and $v_{r}$ are left and right eigenvectors of $A+D$ relative to $\rho(A+D)$.

Proof. Denoting $\Sigma=d I-D$, then the closed loop system is identical to (5), and Theorem 7 applies. In particular, choosing $d=\rho(A+D)$, the closed loop matrix $A+D-d I$ is a singular negated $\mathrm{M}_{\vee}$-matrix and the convergence to $x^{*}$ follows from Theorem 3 .

Although the feedback law (17) uses only local state information, imposing unanimity through Proposition 3 requires to 
check eventual positivity of $A+D$, which is a global property of the graph $\Gamma(A)$.

\section{A. Consensus for eventually positive adjacency matrices}

A standard special case of unanimity is given by the consensus problem [36], in which all agents are required to converge to the same value. Given a signed adjacency matrix $A \in \mathbb{R}^{n \times n}$, let us define the Laplacian of $A$ as the matrix $L \in \mathbb{R}^{n \times n}$ of entries

$$
l_{i k}= \begin{cases}\sum_{j \in \operatorname{adj}(i)} a_{i j} & k=i \\ -a_{i k} & k \neq i .\end{cases}
$$

If we think of a distributed control problem on $\Gamma(A)$ for the system (16), then $L$ can be intended as obtained by choosing $u_{i}=-\sum_{j \in \operatorname{adj}(i)} a_{i j}\left(x_{i}-x_{j}\right)$ [28], [36], or, in matrix form,

$$
\dot{x}=-L x \text {. }
$$

When $\Gamma(A)$ is strongly connected and $A \geq 0, L$ is a singular irreducible M-matrix and the 0 eigenvalue has multiplicity 1. The associated right eigenvector is $\mathbf{1}$, i.e., $x^{*} \in \operatorname{span}(\mathbf{1})$ is a consensus value for (19). When $A$ has negative entries, $L$ defined as in (18) can become unstable, as it is straightforward to verify on examples. In this case, determining conditions guaranteeing the marginal stability of (19) is a difficult task.

Assume first that global quantities such as the spectral radius of $A$ and its right eigenvector are known. Then it is straightforward to obtain the following consensus feedback.

Proposition 4 For any $A \stackrel{\vee}{>} 0$ the system (19) with

$$
L_{1}=\rho(A) V_{r}-A V_{r},
$$

where $\rho(A)$ is the spectral radius of $A$ and $V_{r}=\operatorname{diag}\left(v_{r}\right)$ with $v_{r}>0$ the right eigenvector relative to $\rho(A)$, is such that

$$
x^{*}=\lim _{t \rightarrow \infty} x(t)=\frac{v_{\ell}^{T} x_{o} \mathbf{1}}{v_{\ell}^{T} \mathbf{1}}
$$

where $v_{\ell}$ is the left eigenvector of $A$ relative to $\rho(A)$.

Proof. From Theorem 1, $A \in \mathcal{P F}_{n}$, meaning that $\rho(A) I-A$ is a singular $\mathbf{M}_{\vee}$-matrix. Therefore, from Theorem 3, $\rho(A) I-A$ has $\lambda_{1}(\rho(A) I-A)=0$ of right eigenvector $v_{r}>0$, and $\operatorname{Re}\left[\lambda_{i}(\rho(A) I-A)\right]>0$ for $i=2, \ldots, n$. From $v_{r}=V_{r} \mathbf{1}$, we can rewrite $\rho(A) v_{r}=A v_{r}$ as $\rho(A) V_{r} \mathbf{1}=A V_{r} \mathbf{1}$. Since $v_{r}>0, V_{r}=\operatorname{diag}\left(v_{r}\right)$ is diagonal positive, hence also $L_{1}=$ $\rho(A) V_{r}-A V_{r}$ is a singular $\mathbf{M}_{\vee}$-matrix with $\lambda_{1}\left(L_{1}\right)=0$ and $\operatorname{Re}\left[\lambda_{i}\left(L_{1}\right)\right]>0$ for $i=2, \ldots, n$. By construction, the right eigenvector of $L_{1}$ relative to 0 is now 1 , meaning that $L_{1}$ solves the consensus problem. Convergence to the $x^{*}$ value given in (21) follows from the Perron-Frobenius theorem, after observing that, from Theorem 3 , the left eigenvector of $L_{1}$ relative to 0 is the same $v_{\ell}$ as for $A$ relative to its spectral radius.

For $L_{1}$ as in (20), the expression in coordinates for the feedback is

$$
u_{i}=\rho(A) v_{r, i} x_{i}-\sum_{j \in \operatorname{adj}(i)} a_{i j} v_{r, j} x_{j}, \quad i=1, \ldots, n,
$$

from which the nonlocality of the law is evident, since each node needs to know $\rho(A)$ and $v_{r}$. The following Theorem provides a sufficient condition for the consensus problem of (19) to be solvable in a distributed manner.

Theorem 9 Consider the signed graph $\Gamma(A)$, and define the diagonal matrix $\Sigma=\operatorname{diag}\left(\sigma_{1}, \ldots, \sigma_{n}\right)$, with $\sigma_{i}=$ $\sum_{j \in \operatorname{adj}(i)} a_{i j}, i=1, \ldots, n$. If $\exists$ a scalar $d \geq 0$ such that $A+D>0$, where $D=d I-\Sigma$, then the matrix

$$
L_{2}=\Sigma-A \text {, }
$$

is a singular $M_{\vee}$-matrix, the system (19) holds to $\mathbb{R}_{\{-,+\}}^{n}$ and in particular it converges to

$$
x^{*}=\lim _{t \rightarrow \infty} x(t)=\frac{v_{\ell}^{T} x_{o} \mathbf{1}}{v_{\ell}^{T} \mathbf{1}},
$$

where $v_{\ell}$ is the left eigenvector of $L_{2}$ relative to 0.

Proof. By construction, $L_{2} \mathbf{1}=0$, i.e. 0 is an eigenvalue of $L_{2}$ with 1 the associated right eigenvector. Consider a nonnegative scalar $d$ and define the diagonal matrix $D=d I-\Sigma$. Letting $B=A+D$, then (22) can be rewritten as

$$
L_{2}=\Sigma-A=d I-D-B+D=d I-B .
$$

From $L_{2} \mathbf{1}=0$, it follows that

$$
L_{2} \mathbf{1}=(d I-B) \mathbf{1}=d \mathbf{1}-B \mathbf{1}=0,
$$

i.e., $d$ is an eigenvalue of $B$ with associated eigenvector 1 . Assuming now that $B>0$, then, from Lemma 1, 1 must be its only positive eigenvector and its associated eigenvalue must be the spectral radius of $B: \rho(B)=d$. It follows therefore that $L_{2}=d I-B$ is a singular $\mathbf{M}_{\vee}$-Matrix. From Theorem 3, then, $\lambda_{1}\left(L_{2}\right)=0$ and $\operatorname{Re}\left[\lambda_{i}\left(L_{2}\right)\right]>0$ for $i=2, \ldots, n$, i.e., $L_{2}$ solves the consensus problem and converges to $x^{*}$. From Theorem 7 it also holds to $\mathbb{R}_{\{-,+\}}$.

As with Proposition 3, while the control law is distributed, the eventual positivity of $A+D$ is a global property of $\Gamma(A)$. Notice that in Theorem 9 it is not necessary that $\Sigma \geq 0$, i.e., the adjacency matrix can have negative row sums. Likewise, also $D$ can have negative diagonal entries. It can even happen that $\Sigma$ and $D$ have negative entries simultaneously, see Example 7. Clearly, $d \geq \max _{i}\left(\sigma_{i}\right)$ implies $D \geq 0$.

Example 7 In correspondence of the following signed adjacency matrix:

$$
A=\left[\begin{array}{ccccc}
0 & 0 & -0.25 & -0.15 & 0.3 \\
0 & 0 & 0 & 0 & 2 \\
1 & 0.8 & 0 & 0 & 0 \\
0.5 & 0 & 0.7 & 0 & 0 \\
-0.1 & 0 & 0 & 0.2 & 0
\end{array}\right]
$$

we have $\Sigma=\operatorname{diag}(-0.1,2,1.8,1.2,0.1)$. Choosing $d=1.8$ leads to $D=\operatorname{diag}(1.9,-0.2,0,0.6,1.7)$, i.e., both $\Sigma$ and $D$ have negative entries. $A+D>0$ and $L_{2}=\Sigma-A$ such that $\lambda_{1}\left(L_{2}\right)=0$ and $\operatorname{Re}\left[\lambda_{i}\left(L_{2}\right)\right]>0$ for $i=2, \ldots, 5$. 


\section{B. Stubborn agents as controls}

In this section we consider the following system, similar in structure to (10):

$$
\dot{x}=E x+C u
$$

but in which the stubborn agents may have a time-varying opinion $u(t) \in \mathbb{R}^{r}$ (i.e., they can be assimilated to control inputs). Our aim is to understand to what extent the $u(t)$ can be used to impose unanimity of the $x$ opinions.

A possible way to approach the problem is to make use of the notion of $(E, C)$-invariance from the theory of constrained linear control systems [15]. A cone $\mathcal{K}=\operatorname{cone}(\Omega)$ is said $(E, C)$-invariant for a system like (23) if $\forall x_{o} \in \mathcal{K} \exists$ a function $u(t) \in \mathbb{R}^{r}$ such that $x(t) \in \mathcal{K} \forall t \geq 0$. A necessary and sufficient condition for $(E, C)$-invariance is the following proposition, adapted from [15] (see also [7], [31]).

Proposition 5 Consider the system (23) and a cone $\mathcal{K}=$ cone $(\Omega), \Omega \in \mathbb{R}^{n \times \mu} . \mathcal{K}$ is $(E, C)$-invariant for (23) if and only if $\exists$ a matrix $U \in \mathbb{R}^{r \times \mu}$ and an essentially nonnegative matrix $H \in \mathbb{R}^{\mu \times \mu}$ such that

$$
E \Omega+C U=\Omega H
$$

Since the columns of $\Omega$ represent the generating vectors of $\mathcal{K}$, the interpretation of the condition (24) is that if at each vertex $\omega_{i}$ of $\mathcal{K} \exists$ a value $u_{i}$ such that $E x_{i}+C u_{i} \in \mathcal{K}$ (subtangentiality condition), then a time-varying $u(t)$ rendering $\mathcal{K}$ invariant for (23) can be found, and viceversa.

This condition, however, is incompatible with the scenario of totally stubborn agents described in Section III-D. Even in the case in which the same constant control can be used for all vertices, without eventual positivity on $E$ (or on $B=E+d I$ ), unanimity cannot be guaranteed (see Example 8).

If we assume further that the stubborn agents have full information on the state $x$, then a feedback design $u=\Phi(x)$ can be sought. The problem is then closely related to a linear constraint feedback stabilization problem [7]. However, understanding in which cases it is possible to achieve eventual positivity through linear feedback remains an open question.

Example 8 Let us consider

$$
E=A-\Sigma=\left[\begin{array}{ccc}
-0.969 & 0.143 & 1.7 \\
-0.352 & -1.182 & 0.2 \\
0 & 0.268 & 0.09
\end{array}\right]
$$

and

$$
C=\left[\begin{array}{c}
-0.11 \\
0.36 \\
0
\end{array}\right]
$$

Notice that $\nexists d \in \mathbb{R}$ such that $B=A+D \stackrel{\vee}{>} 0$, where $D=d I-\Sigma$. Consider as cone $\mathcal{K}=\mathbb{R}_{+}^{3}$, which corresponds to choosing $\Omega=I_{3}$. Then the $(E, C)$-invariance condition (24) amounts to solvability on

$$
E+C\left[\begin{array}{lll}
u_{1} & u_{2} & u_{3}
\end{array}\right]=H
$$

for $H$ essentially nonnegative. In this case, even a solution with all equal $u_{i}$ exists: $u_{1}=u_{2}=u_{3}=0.98$. However, if we consider the "open loop" system (23) with the constant control $u=u_{1}$, then unanimity is not preserved, even if initial conditions are unanimous, see Fig. 5 (a). If instead we implement a state feedback law, then holdability to $\mathbb{R}_{\{+,-\}}^{3}$ can be easily imposed. In this case, since "subtangentiality", [7] can be achieved by the same control at all vertices, a linear state feedback is easily found: $u=u_{1} x$. It can be checked that $E+u_{1} C\left[\begin{array}{lll}1 & 1 & 1\end{array}\right]+d I \stackrel{\vee}{>} 0$, where e.g. $d=1$, although the resulting closed-loop is unstable, see Fig. 5 (b).

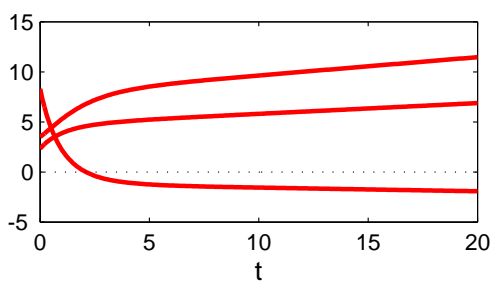

(a)

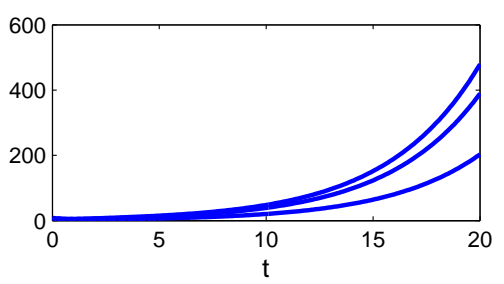

(b)

Fig. 5. Example 8. (a): The system (23) driven by the value $u=u_{1}$ of the totally stubborn agent which fulfills Proposition 5 does not achieve unanimity. (b): If instead of a constant $u$ we use a state feedback $u=u_{1} x$, then holdability to $\mathbb{R}_{\{+,-\}}^{3}$ is obtained.

\section{HOLDABLE BIPARTITE OPINIONS}

In this Section the notion of eventual positivity is combined with the idea of structural balance through the orthant transformations (or gauge transformations) that the latter entails [4].

Associated with $s \in \mathcal{S}$ is the following partial order relationship for $\mathbb{R}^{n}$ vectors (indicated " $\geq_{s}$ "): $x_{1} \geq_{s} x_{2}$ if and only if $S x_{1} \geq S x_{2}$ where $S=\operatorname{diag}(s)$. Similarly: $x_{1}>_{s} x_{2}$ if and only if $S x_{1}>S x_{2}$. The change from standard ordering (given by the partial order vector 1) to the partial ordering given by any $s$ is performed by what we call a gauge transformation:

$$
A \rightarrow S A S,
$$

see [4] for the details. The effect of a similarity transformation such as (25) is to change sign to all rows and columns for which $s_{i}=-1$. It is possible to use the notion of gauge transformation (25) to extend the results presented above from predictable unanimous opinions to predictable non-unanimous opinions.

Definition $6 A \in \mathbb{R}^{n \times n}$ has the signed strong PerronFrobenius property if the spectral radius $\rho(A)$ is a real positive eigenvalue of $A$ that is strictly larger in modulus than any 
other eigenvalue of $A$, and its right eigenvector $v_{r}$ is such that $v_{r, i} \neq 0 \forall i=1, \ldots, n$.

Denoting $\mathcal{S P} \mathcal{F}_{n}$ the set of matrices in $\mathbb{R}^{n \times n}$ possessing the signed strong Perron-Frobenius property, then when both $A \in \mathcal{S P} \mathcal{F}_{n}$ and $A^{T} \in \mathcal{S} \mathcal{P} \mathcal{F}_{n}$ and the components of $v_{\ell}$ and $v_{r}$ have exactly the same sign pattern (or exactly the opposite sign pattern) we have the following.

Proposition 6 Given $A \in \mathbb{R}^{n \times n}$, then the following are equivalent:

1) $A \in \mathcal{S P F} \mathcal{F}_{n}, A^{T} \in \mathcal{S P} \mathcal{F}_{n}$, and the left and right eigenvectors of $A$ are such that $v_{\ell, i} v_{r, i}>0 \forall i=1, \ldots, n$, or $v_{\ell, i} v_{r, i}<0 \forall i=1, \ldots, n$;

2) $\exists s \in \mathcal{S}$ such that $S A S \stackrel{\vee}{>} 0$, where $S=\operatorname{diag}(s)$.

Proof. The first condition implies that the right and left eigenvectors of $A$ associated to $\rho(A)$ have the same sign pattern $^{1}$. This means $\exists s \in \mathcal{S}$ such that $v_{s, r}=S v_{r}>0$ and $v_{s, \ell}=S v_{\ell}>0$. But then from $A v_{r}=\rho(A) v_{r}$ we can write

$$
S A v_{r}=\rho(A) S v_{r}
$$

or, since $S^{2}=I$,

$$
S A S v_{s, r}=\rho(A) v_{s, r},
$$

which implies that $A_{s}=S A S \in \mathcal{P} \mathcal{F}_{n}$ since $\operatorname{sp}(A)=\operatorname{sp}\left(A_{s}\right)$ and $v_{s, r}>0$ is the right eigenvector relative to the spectral radius $\rho\left(A_{s}\right)$. Analogously, $A_{s}^{T} \in \mathcal{P} \mathcal{F}_{n}$, hence from Theorem $1, A_{s} \stackrel{\vee}{>} 0$. The opposite implication follows by the same argument.

Example 9 The matrix

$$
A_{1}=S A S=\left[\begin{array}{cccc}
0 & 0 & 0 & -39 \\
0 & 0 & -92 & 9 \\
0 & -117 & 0 & 50 \\
-5 & 0 & -111 & 0
\end{array}\right]
$$

is the gauge transformation of Example 1, with $s=$ $\left[\begin{array}{llll}1 & -1 & 1 & -1\end{array}\right]$.

Checking the signed strong Perron-Frobenius property is much more difficult that checking if $A \stackrel{\vee}{>} 0$. The problem is equivalent to a MAX-CUT problem (or one of its equivalent problems, see [24], [18] for an overview) and it is known to be NP-hard. Proposition 6 suggests a possible algorithm, namely checking if $A$ is gauge equivalent to an eventually positive matrix. As can be seen comparing Examples 1 and 9, normally the rows and columns in correspondence of the -1 entries of $s$ have a negative sum, while in the gauge transformed matrix these sums are positive. Notice that this rule of thumb need not be strictly observed always (see Example 7 for a counterexample). It however provides a useful heuristic procedure for gauge transforming $A$ into a matrix more likely

\footnotetext{
${ }^{1}$ When they have exactly the opposite sign pattern, the argument is identical up to a trivial modification.
}

to be eventually positive. The following algorithm is inspired by [24].

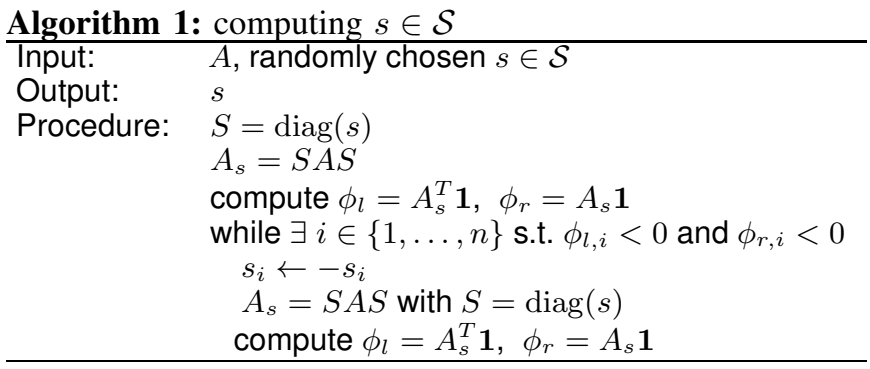

The partial order $s$ returned by Algorithm 1 is the gauge transformation sought in Proposition 6. Since Algorithm 1 can terminate in a local optimum, it is useful to run it repeatedly, randomly changing the initial $s \in \mathcal{S}$.

Theorem 10 Consider the system (5). If $\exists d \in \mathbb{R}$ such that Proposition 6 holds for $A+D$, where $D=d I-\Sigma$, then the system (5) holds to the orthant pair $\mathbb{R}_{\{-s, s\}}^{n}$ for some $s \in \mathcal{S}$.

Proof. Denote $B=A+D \in \mathcal{S} \mathcal{P} \mathcal{F}_{n}, B^{T} \in \mathcal{S} \mathcal{P} \mathcal{F}_{n}$, with identical sign pattern for $v_{\ell}$ and $v_{r}$. Then, from Proposition 6, $\exists s \in \mathcal{S}$ such that $B_{s}=S B S \stackrel{\vee}{>} 0, S=\operatorname{diag}(s)$. From Theorem 7, calling $z=S x$, the system

$$
\dot{z}=-S(\Sigma-A) S z=-\left(d I-B_{s}\right) z
$$

holds to the orthant pair $\mathbb{R}_{\{-,+\}}^{n}$, which implies that $x=S z$ must hold to $\mathbb{R}_{\{-s, s\}}^{n}$.

Example 10 Large scale example similar to Example 3: $n=$ 100 agents connected through $m=1000$ randomly chosen edges, of which 527 are negative and 473 positive. The 3 cases shown in Fig. 6 are qualitatively analogous to those of Fig. 2. In all 3 cases the system holds to $\mathbb{R}_{\{-s, s\}}^{n}$ for some $s \in \mathcal{S}$.

Analogously to Corollary 3, the sufficient condition of the previous Theorem can be weakened to a "one-side" signed Perron-Frobenius condition, and reformulated in terms of invariant cones fully contained in one of the orthants of $\mathbb{R}^{n}$.

Corollary 4 Consider the system (5). If $\exists d \in \mathbb{R}$ such that for $D=d I-\Sigma, A+D \in \mathcal{S P \mathcal { F }}$, then the system (5) holds to the orthant pair $\mathbb{R}_{\{-s, s\}}^{n}$ for some $s \in \mathcal{S}$.

Proof. The proof is straightforward, from Corollary 2, and the proof of Corollary 3.

\section{ACHIEVING UNANIMITY IN DISCRETE-TIME SYSTEMS}

In general, a linear discrete-time distributed process of opinion forming will be given by the system

$$
x(k+1)=W x(k), \quad W=\Delta+F,
$$

where we assume $F$ off-diagonal and $\Delta=\operatorname{diag}\left(\delta_{1}, \ldots, \delta_{n}\right)$, $\delta_{i}>0$, i.e., nodes never change their opinions only based on their current state. The analogous of Theorem 6 is the 


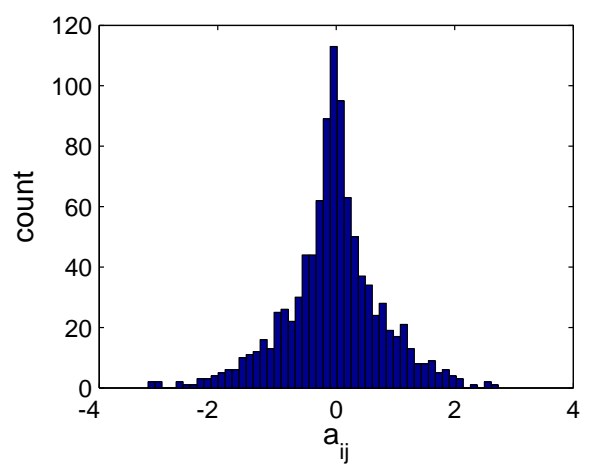

(a)
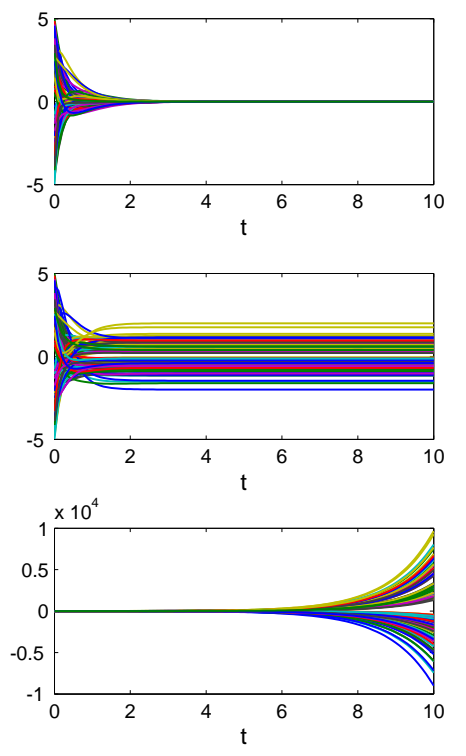

(b)

Fig. 6. Example 10. In (a) the distribution of the nonzero edges of $A$ is shown. In (b), from top to bottom, the asymptotically stable case, the marginally stable case and the unstable case are shown.

following theorem, whose proof is omitted as it follows the same steps of its continuous-time counterpart.

Theorem 11 Consider the system (27), with $F \stackrel{\vee}{>} 0$.

(i) If $\delta_{i} \leq 1-\rho(F), \forall i=1, \ldots, n$, and $\exists$ at least one $\delta_{i}$ such that $\delta_{i}<1-\rho(F)$, then $x^{*}=\lim _{k \rightarrow \infty} x(k)=0$;

(ii) If $\delta_{i}=1-\rho(F), \forall i=1, \ldots, n$, then $x^{*}=$ $\frac{1}{v_{\ell}^{T} v_{r}} v_{\ell}^{T} x_{o} v_{r}$, where $v_{\ell}$ and $v_{r}$ are respectively the left and right eigenvector of $F$ associated to $\rho(F)$, and $x_{o} \in \mathbb{R}^{n}$ the initial condition;

(iii) If $\delta_{i} \geq 1-\rho(F), \forall i=1, \ldots, n$, and $\exists$ at least one $\delta_{i}$ such that $\delta_{i}>1-\rho(F)$, then $x^{*}= \pm \infty$.

\section{A. Holdability of an opinion}

Theorem 12 Consider the system (27), with $W=\Delta+F$, $\Delta=\operatorname{diag}\left(\delta_{1}, \ldots, \delta_{n}\right), \delta_{i}>0$. If $\exists d \geq 0$ such that $F+D>0$, where $D=\Delta-d I$, then the system (27) holds to $\mathbb{R}_{\{-,+\}}^{n}$.

Proof. Calling $G=F+D$, let us write $W$ as

$$
W=\Delta+F=d I+D+F=d I+G .
$$

Since $G \stackrel{\vee}{>} 0, \rho(G)$ is a strictly dominating positive real eigenvalue of $G$. If $v_{\ell}>0$ and $v_{r}>0$ are its left and right eigenvectors, then $d>0$ implies that $W$ must have $d+\rho(G)$ as eigenvalue of largest modulus, with the same $v_{\ell}$ and $v_{r}$ as eigenvectors. It follows therefore that $W, W^{T} \in \mathcal{P} \mathcal{F}_{n}$ and, from Theorem 1 , that $W \stackrel{\vee}{>} 0$. Hence

$$
\lim _{k \rightarrow \infty}(d I+G)^{k} /(d+\rho(G))^{k}=v_{r} v_{\ell}^{T} / v_{\ell}^{T} v_{r}
$$

and

$$
x^{*}=\lim _{k \rightarrow \infty} x(k)=v_{\ell}^{T} x_{o} v_{r} / v_{\ell}^{T} v_{r} .
$$

If $v_{\ell}^{T} x_{o}>0$ then $x^{*} \in \operatorname{int}\left(\mathbb{R}_{+}^{n}\right) \cup \emptyset$, while if $v_{\ell}^{T} x_{o}<0$ then $x^{*} \in \operatorname{int}\left(\mathbb{R}_{-}^{n}\right) \cup \emptyset$. Assuming we are in the first case, from Theorem $4, W \stackrel{\vee}{>} 0$ implies that $\exists$ a $W$-invariant convex cone $\mathcal{K}=\operatorname{cone}(\Omega), \Omega \in \mathbb{R}^{n \times \mu}$ full row rank, such that $\mathcal{K} \subset$ $\operatorname{int}\left(\mathbb{R}_{+}^{n}\right) \cup \emptyset$ and for which $W$ is $\mathcal{K}$-positive. Recalling that for discrete-time systems such as (27) a necessary and sufficient condition for invariance is the existence of $H \in \mathbb{R}_{+}^{\mu \times \mu}$ such that $W \Omega=\Omega H$ [19], the proof is now analogous to that of Theorem 7 .

\section{B. Recovering positivity through (down)sampling}

The following theorem says that whenever a continuoustime system has the eventual positivity property, then provided the sampling time is sufficiently long, it admits an exact discretization in which the contribution of the negative entries has disappeared.

Theorem 13 Consider the continuous-time system (5), with $E=A-\Sigma$. Assume $\exists d \in \mathbb{R}$ such that $A+D \stackrel{\vee}{>} 0$, where $D=d I-\Sigma$. Then $\exists$ a sampling time $\tau_{o}>0$ such that for $\tau \geq \tau_{o}$ the discretized system

$$
z(k+1)=F z(k)
$$

where $z(k)=x(\tau k)$ and $F=e^{\tau E}$, is such that $F>0$. The system (29) holds to the orthant pair $\mathbb{R}_{\{-,+\}}$.

Proof. From Lemma $2, B=A+D \stackrel{\vee}{>} 0$ implies that $E=$ $B-d I$ is eventually exponentially positive, i.e., that $e^{\tau E}>0$ $\forall \tau \geq \tau_{o}$, where $\tau_{o}$ is the exponential index of $E$.

Clearly $F>0$ implies that the discretized system (29) is not distributed, as it evolves on a fully connected graph, not on the original $\Gamma(A)$.

An analogous downsampling is possible for the system (27), when $W \stackrel{\vee}{>} 0$.

\section{A special case: eventually stochastic matrices}

A common use of discrete-time nonnegative systems is as transition probabilities in Markov chains [37]. In this case $W \geq 0$ is chosen to be a stochastic matrix. A matrix $W$ is column stochastic if $\mathbf{1}^{T} W=\mathbf{1}^{T}, 0 \leq w_{i j} \leq 1$, meaning that $\mathbf{1}$ is a left eigenvector associated to $\rho(W)=1$. Analogously, $W$ is row stochastic if $W \mathbf{1}=\mathbf{1}, 0 \leq w_{i j} \leq 1$, and doubly stochastic if it is both row and column stochastic. 
Definition 7 A matrix $W$ is said eventually column (resp. row) stochastic if $W \stackrel{\vee}{>} 0$ and $\mathbf{1}^{T} W=\mathbf{1}^{T}($ resp. $W \mathbf{1}=\mathbf{1})$. It is said eventually doubly stochastic if it is both eventually row and column stochastic.

Lemma 3 If $W$ is eventually row (or column) stochastic, then $\rho(W)=1$ is a positive eigenvalue of $W$.

Proof. From Theorem 1, W, $W^{T} \in \mathcal{P} \mathcal{F}_{n}$, hence $\rho(W)$ is a positive eigenvalue of positive left and right eigenvectors $v_{\ell}$ and $v_{r}$. Lemma 1 implies that for $W \stackrel{\vee}{>} 0$, the positive eigenvectors must be unique up to a scalar constant, hence it must be $v_{r}=\alpha \mathbf{1}$ if $W$ is eventually row stochastic (or $v_{\ell}=\alpha \mathbf{1}$ if $W$ is eventually column stochastic), $\alpha \in \mathbb{R}$. It follows that $\rho(W)=1$.

Clearly when $W$ is not nonnegative then any probabilistic interpretation associated to the state in (27) is lost. However, since $W^{k}>0$ for $k \geq k_{o}$, any sufficiently long downsampling of the system (27) can still be considered a well-posed transition matrix, provided $W$ is eventually stochastic.

\section{Application: PageRank with negative links}

PageRank, the algorithm at the basis of Google search engine provides a measure of importance of web pages based on the number of incoming hyperlinks from other web pages and based on the importance that these other web pages have. It relies on computing the dominant eigenvector of a stochastic matrix of hyperlinks. As long as only positive links are considered, all components of the Perron-Frobenius eigenvector are nonnegative and represent probabilities, hence can be used as a measure of authority and provide a ranking of the web pages.

Let $Q \in \mathbb{R}^{n \times n}$ be the signed adjacency matrix of weblinks, $q_{i j}=\{-1,0,+1\}$. To deal with negative edges, call $p_{j}$ and $n_{j}$ the number of outgoing positive and negative links at node $j=1, \ldots, n$. We assume for the sake of simplicity that $c_{j}=$ $p_{j}-n_{j}=\sum_{i} q_{i j} \neq 0 \forall j=1, \ldots, n$ (which implies that $p_{j}+n_{j} \neq 0 \forall j$, i.e., that there are no dangling nodes [25] $)^{2}$. The normalized signed adjacency matrix is then $A \in \mathbb{R}^{n \times n}$ of entries

$$
a_{i j}= \begin{cases}\frac{\operatorname{sgn}\left(q_{i j}\right)}{c_{j}} & \text { if } j \in \operatorname{adj}(i) \\ 0 & \text { otherwise. }\end{cases}
$$

By construction, then, $\mathbf{1}^{T} A=\mathbf{1}$ and 1 is an eigenvalue of $A$. Since $A$ need not be strongly connected, the condition of eventual positivity cannot be applied in this context, although eventual nonnegativity can. If $A \stackrel{\vee}{\geq}$, then the spectral radius $\rho(A)$ is a positive eigenvalue, although it may not strictly dominate all other eigenvalues of $A$. Following the procedure described e.g. in [10], [25], let us consider the modified matrix

$$
W=(1-m) A+\frac{m}{n} \mathbf{1 1}^{T}, \quad m \in(0,1) .
$$

Similarly to the nonnegative case, it is possible to use (30) to compute the PageRank eigenvector $x^{*}$, via the power method

\footnotetext{
${ }^{2}$ Notice that the degenerate cases $c_{j}=0$ could be also easily bypassed by weighting unevenly positive and negative edges.
}

on the modified problem

$$
x(k+1)=W x(k)=\left((1-m) A+\frac{m}{n} \mathbf{1 1}^{T}\right) x(k) .
$$

Proposition 7 If $A \stackrel{\vee}{\geq} 0$ then $W^{T} \in \mathcal{P} \mathcal{F}_{n} \forall m \in(0,1)$.

Proof. Since by construction 1 is a left eigenvector of $A, A$ and $W$ differ by a rank-1 matrix multiple of this eigenvector, hence Theorem 2.10 of [33] applies, which implies $\rho(W)>$ $\rho(A)>0$. Since 1 is a left eigenvector also for $W$, the claim follows.

In spite of Proposition 7, $A \stackrel{\vee}{\geq} 0 \not \Longrightarrow W \stackrel{\vee}{\geq} 0$, as Example 5 shows. Clearly, if $\min \left(c_{j}\right)>\frac{m}{(1-m) n}$ then $W>0$, i.e., small fractions of negative links are irrelevant and disappear in the power iteration (31). If $W \ngtr 0$ but $W \stackrel{\vee}{>} 0$, then we have the following.

$$
\begin{aligned}
& \text { Theorem } 14 \text { If } W \stackrel{\vee}{>} 0 \text {, and } x(0) \text { obeys } \\
& \qquad 0 \leq x_{i}(0) \leq 1, \quad \mathbf{1}^{T} x(0)=1,
\end{aligned}
$$

then the system (31) holds to $\mathbb{R}_{+}^{n}$ and converges to a $x^{*}$ such that $0 \leq x_{i}^{*} \leq 1, \mathbf{1}^{T} x^{*}=1$.

Proof. From $1^{T} A=1$, one has

$\mathbf{1}^{T} W=(1-m) \mathbf{1}^{T} A+\frac{m}{n} \mathbf{1}^{T} \mathbf{1 1}^{T}=(1-m) \mathbf{1}^{T}+m \mathbf{1}^{T}=\mathbf{1}^{T}$

i.e., $W$ is an eventually column stochastic matrix. Therefore, from Lemma 3, $\rho(W)=1$, and $\mathbf{1}^{T} x(k+1)=\mathbf{1}^{T} W x(k)=$ $\mathbf{1}^{T} x(k)$, i.e., $\mathbf{1}^{T} x(k)=1 \forall k$ when (32) holds. Holdability to $\mathbb{R}_{+}^{n}$ follows from Theorem 12 with $d=0$.

Notice that even if $x(0) \geq 0$ and $x^{*} \geq 0, x(k)$ may have negative entries during the transient. Introducing a discretetime analogue of Corollary 4, it is possible to extend the method to "signed ranking" (i.e., to include negative reputations in the final ranks).

Proposition 8 Assume $W \in \mathcal{S P \mathcal { F }}$ and that $x(0)$ obeys (32). Then the system (31) holds to $\mathbb{R}_{\{-s, s\}}^{n}$ for some $s \in \mathcal{S}$, and converges to a $x^{*}$ such that $1^{T} x^{*}=1$ and $\operatorname{sgn}\left(x^{*}\right)=$ $\pm \operatorname{sgn}(s)$.

Proof. The first part follows from Corollary 4. The second part from an expression like (28) in which $v_{\ell}=1$ and $v_{\ell}^{T} x_{o}=1$, meaning that $x^{*}=v_{r} / \mathbf{1}^{T} v_{r}$.

\section{CONCLUSION}

Being able to predict or influence the outcome of an opinion forming process is an important problem in social network theory. However, even for linear dynamics, this becomes a difficult task as soon as non-cooperative interactions are taken into account (understanding the cooperative case, on the contrary, is trivial). In this paper we have shown how the PerronFrobenius theorem can be used for this task also beyond its standard formulation for cooperative systems. In particular we have shown how it is possible to associate the achievement of holdable opinions with the existence of invariant cones, 
and the achievement of holdable unanimous opinions with invariant cones properly contained in the positive orthant. These cases correspond to signed adjacency matrices having the eventual positivity property, i.e., such that in sufficiently high powers all negative entries have disappeared. For them the effect of the negative influences is only transient and does not alter the asymptotic behavior of the system. However, unlike e.g. structural balance, eventual positivity is not a graphical property obeyed by all signed graphs having the same sign pattern. It is on the contrary dependent on the numerical entries of the adjacency matrix, and this is reflected by the lack of convexity of the set of eventually positive matrices, as shown in our examples. Nevertheless, the eventual positive characterization and its geometric interpretation are useful to disentangle the behavior of dynamical networks having antagonistic interactions. The perspective of invariant cones is useful also to understand how a network behaves in presence of stubborn agents, and how these last may provide an opportunity for control of opinion dynamics on networks. Finally, although in this paper we considered only linear deterministic models, we expect that also more realistic opinion dynamics models such as for example gossip and other randomized strategies [21] will show similar properties, in the same way as it happens for structural balance when passing from deterministic models [4] to gossiping schemes [38].

\section{ACKNOWLEDGMENTS}

The authors would like to thank the reviewers for a careful reading of a preliminary version of the manuscript.

\section{APPENDIX}

\section{Proof of Theorem 6}

From Theorem 1, since $A \in \mathcal{P} \mathcal{F}_{n}$, its spectral radius $\rho(A)$ is a simple real positive eigenvalue of $A$ with positive left and right eigenvectors $v_{\ell}$ and $v_{r}$, and these are the only positive eigenvectors of $A$. Defining the diagonal matrix $\Xi=\Sigma-$ $\rho(A) I$, then it is possible to rewrite the matrix $E$ as follows

$$
E=A-\Xi-\rho(A) I \text {. }
$$

Consider the case $\sigma_{i} \geq \rho(A), \forall i=1, \ldots, n$, with $\sigma_{i}>\rho(A)$ for at least an index $i$. Then $E$ is given by the sum of the singular negated $\mathbf{M}_{\vee}$-matrix $A-\rho(A) I$ and of the nonpositive diagonal matrix $-\Xi$. From Theorem 3, $\operatorname{Re}\left[\lambda_{i}(\rho(A) I-A)\right] \geq 0$, and $\lambda_{1}(\rho(A) I-A)=0$ has multiplicity 1 . Since $\operatorname{Re}\left[\lambda_{i}(\Xi)\right] \geq 0$, it must be

$$
\operatorname{Re}\left[\lambda_{i}(E)\right] \leq \operatorname{Re}\left[\lambda_{i}(A-\rho(A) I)\right] \leq 0 .
$$

The only possibility for $E$ to be singular is that $\Xi$ and $A-\rho(A) I$ have both 0 as eigenvalue with the same eigenvector, i.e. that $\operatorname{Ker}(A-\rho(A) I) \bigcap \operatorname{Ker}(\Xi) \neq \emptyset$. But since $\operatorname{Ker}(A-\rho(A) I)=\operatorname{span}\left(v_{r}\right)$ and $v_{r}>0$, it must be $\Xi v_{r} \neq 0$ always when $\Xi$ has at least a positive diagonal entry. Hence $\operatorname{Re}\left[\lambda_{i}(E)\right]<0, i=1, \ldots, n$, and $E$ is asymptotically stable.

If $\sigma_{i}=\rho(A), \forall i=1, \ldots, n$, then $\Xi=0$ and $E=$ $A-\rho(A) I$ is a singular negated $\mathbf{M}_{\vee}$-matrix, for which the following relations hold

$$
E v_{r}=(A-\rho(A) I) v_{r}=A v_{r}-\rho(A) v_{r}=0,
$$

and

$$
v_{\ell}^{T} E=v_{\ell}^{T}(A-\rho(A) I)=v_{\ell}^{T} A-v_{\ell}^{T} \rho(A)=0 .
$$

Then 0 is a simple eigenvalue of $E$ with associated left and right eigenvectors $v_{\ell}$ and $v_{r}$ respectively. From Theorem 3, all the other eigenvalues of $E$ are such that $\operatorname{Re}\left[\lambda_{i}(E)\right]<0$, for $i=2, \ldots, n$, meaning that $E$ is marginally stable. It follows that

$$
x^{*}=\lim _{t \rightarrow+\infty} x(t)=\lim _{t \rightarrow+\infty} e^{E t} x_{o}=\frac{1}{v_{\ell}^{T} v_{r}} v_{\ell}^{T} x_{o} v_{r},
$$

and the second item of the theorem is proved.

Finally, if $\sigma_{i} \leq \rho(A), \forall i=1, \ldots, n$, and $\exists$ at least one $\sigma_{i}$ such that $\sigma_{i}<\rho(A)$, then the matrix $\Xi=\operatorname{diag}\left(\xi_{1}, \ldots, \xi_{n}\right)=$ $\operatorname{diag}\left(\sigma_{1}-\rho(A), \ldots, \sigma_{n}-\rho(A)\right)$ is nonzero and nonpositive. Consider the projection of the dynamics (5) on the left eigenspace of $\rho(A)$ :

$$
\begin{aligned}
\frac{d}{d t}\left\langle v_{\ell}, x\right\rangle & =\left\langle v_{\ell}, \dot{x}\right\rangle=v_{\ell}^{T}(A-\Xi-\rho(A) I) x \\
& =-v_{\ell}^{T} \Xi x=-\left[\begin{array}{lll}
v_{\ell, 1} \xi_{1} & \ldots & v_{\ell, n} \xi_{n}
\end{array}\right] x .
\end{aligned}
$$

Since $v_{\ell}>0, \xi_{i} \leq 0$ and $\xi_{i}<0$ for at least an index $i$, when $x \in \operatorname{int}\left(\mathbb{R}_{+}^{n}\right)$ we have:

$$
\left\langle v_{\ell}, \dot{x}\right\rangle=-\sum_{i=1}^{n} v_{\ell, i} \xi_{i} x_{i}>0,
$$

meaning that the projection grows unbounded, i.e., that the system (5) must be unstable.

\section{REFERENCES}

[1] D. Acemoglu, G. Como, F. Fagnani, and A. Ozdaglar. Opinion fluctuations and persistent disagreement in social networks. In Decision and Control and European Control Conference (CDC-ECC), 2011 50th IEEE Conference on, pages 2347-2352, 2011.

[2] Daron Acemoglu and Asuman Ozdaglar. Opinion dynamics and learning in social networks. Dynamic Games and Applications, 1(1):3-49, 2011.

[3] C. Altafini. Dynamics of opinion forming in structurally balanced social networks. PLoS ONE, 7(6):e38135, 2012.

[4] C. Altafini. Consensus problems on networks with antagonistic interactions. IEEE Transactions on Automatic Control, 58(4):935-946, 2013.

[5] A. Berman and R.J. Plemmons. Nonnegative matrices in the mathematical sciences. Classics in applied mathematics. Society for Industrial and Applied Mathematics, 1994.

[6] F. Blanchini. Set invariance in control. Automatica, 35:1747-1767, 1999.

[7] F. Blanchini and S. Miani. Set-Theoretic Methods in Control. Systems \& control. Birkhäuser Boston, 2008.

[8] V.D. Blondel, J.M. Hendrickx, and J.N. Tsitsiklis. On Krause's multiagent consensus model with state-dependent connectivity. Automatic Control, IEEE Transactions on, 54(11):2586 -2597, nov. 2009.

[9] Sergey Brin and Lawrence Page. The anatomy of a large-scale hypertextual web search engine. In Proceedings of the seventh international conference on World Wide Web 7, WWW7, pages 107-117, Amsterdam, The Netherlands, 1998. Elsevier Science Publishers B. V.

[10] K. Bryan and T. Leise. The $\$ 25,000,000,000$ eigenvector: The linear algebra behind Google. SIAM Review, 48(3):569-581, 2006.

[11] D. Cartwright and F. Harary. Structural balance: a generalization of Heider's theory. Psychological Review, 63:277-292, 1956.

[12] E.B. Castelan and J.-C. Hennet. On invariant polyhedra of continuoustime linear systems. Automatic Control, IEEE Transactions on, 38(11):1680-1685, 1993.

[13] Fan Chung, Alexander Tsiatas, and Wensong Xu. Dirichlet PageRank and ranking algorithms based on trust and distrust. Internet Mathematics, 9(1):113-134, 2013. 
[14] C. De Kerchove and P. Van Dooren. The PageTrust algorithm: how to rank web pages when negative links are allowed. In Proc. SIAM Int. Conf. on Data Mining, pages 346-252, 2008.

[15] C.E. T. Dórea and J.-C. Hennet. (A, B)-invariance conditions of polyhedral domains for continuous-time systems. European Journal of Control, 5(1):70 - 81, 1999.

[16] David Easley and Jon Kleinberg. Networks, Crowds, and Markets. Reasoning About a Highly Connected World. Cambridge Univ. Press, Cambridge, 2010.

[17] Abed Elhashash and Daniel B. Szyld. On general matrices having the Perron-Frobenius property. ELA. The Electronic Journal of Linear Algebra [electronic only], 17:389-413, 2008.

[18] G. Facchetti, G. Iacono, and C. Altafini. Computing global structural balance in large-scale signed social networks. Proc. Nat. Ac. Sci., 108:20953-8, 2011

[19] L. Farina and L. Benvenuti. Invariant polytopes of linear systems. IMA Journal of Mathematical Control and Information, 15(3):233-240, 1998.

[20] L. Farina and S. Rinaldi. Positive Linear Systems: Theory and Applications. A Wiley-Interscience publication. Wiley, 2000.

[21] Paolo Frasca, Chiara Ravazzi, Roberto Tempo, and Hideaki Ishii. Gossips and prejudices: ergodic randomized dynamics in social networks. In 4th IFAC Workshop on Distributed Estimation and Control in Networked Systems, volume 4, pages 212-219. International federation of Automatic Control, September 2013.

[22] Noah E Friedkin and Eugene C Johnsen. Social influence networks and opinion change. In E. J. Lawler and M. W. Macy, editors, Advances in Group Processes, volume 16, pages 1-29. 1999.

[23] Shmuel Friedland. On an inverse problem for nonnegative and eventually nonnegative matrices. Israel Journal of Mathematics, 29(1):43-60, 1978.

[24] Giovanni Iacono, Fahimeh Ramezani, Nicola Soranzo, and Claudio Altafini. Determining the distance to monotonicity of a biological network: a graph-theoretical approach. IET Syst. Biol., 4(3):223-235, 2010.

[25] H. Ishii and R. Tempo. Distributed randomized algorithms for the PageRank computation. Automatic Control, IEEE Transactions on, 55(9):1987-2002, 2010.

[26] Charles R. Johnson and Pablo Tarazaga. On matrices with PerronFrobenius properties and some negative entries. Positivity, 8(4):327-338, 2004.

[27] John Maybee and James Quirk. Qualitative problems in matrix theory. SIAM Review, 11(1):pp. 30-51, 1969.

[28] M. Mesbahi and M. Egerstedt. Graph-theoretic Methods in Multiagent Networks. Princeton University Press, 2010.

[29] Basílio E.A. Milani and Carlos E.T. Dórea. On invariant polyhedra of continuous-time systems subject to additive disturbances. Automatica, 32(5):785 - 789, 1996.

[30] I.-C. Morarescu and A. Girard. Opinion dynamics with decaying confidence: Application to community detection in graphs. Automatic Control, IEEE Transactions on, 56(8):1862-1873, 2011.

[31] Fabio Morbidi, Francesco Bullo, and Domenico Prattichizzo. Visibility maintenance via controlled invariance for leader-follower vehicle formations. Automatica, 47(5): 1060 - 1067, 2011.

[32] D. Noutsos and M. Tsatsomeros. Reachability and holdability of nonnegative states. SIAM Journal on Matrix Analysis and Applications, 30(2):700-712, 2008.

[33] Dimitrios Noutsos. On Perron-Frobenius property of matrices having some negative entries. Linear Algebra and its Applications, 412:132 153,2006

[34] Dale D. Olesky, Michael J. Tsatsomeros, and Pauline van den Driessche. $\mathrm{M}_{\vee}$-matrices: a generalization of M-matrices based on eventually nonnegative matrices. ELA. The Electronic Journal of Linear Algebra, 18:339-351, 2009.

[35] N. J. Pullman. A geometric approach to the theory of nonnegative matrices. Linear Algebra and Appl., 4:297-312, 1971.

[36] Wei Ren, R.W. Beard, and E.M. Atkins. Information consensus in multivehicle cooperative control. Control Systems, IEEE, 27(2):71 -82, 2007.

[37] E. Seneta. Non-negative Matrices and Markov Chains. Springer Series in Statistics. Springer, 2006.

[38] Guodong Shi, Alexandre Proutiere, Mikael Johansson, John Baras, Karl $\mathrm{H}$ Johansson, et al. Emergent behaviors over signed random networks in dynamical environments. arXiv preprint arXiv:1309.5488, 2013.

[39] Hal L. Smith. Systems of ordinary differential equations which generate an order preserving flow. A survey of results. SIAM Rev., 30(1):87-113, 1988.
[40] S. Tarbouriech and C. Burgat. Positively invariant sets for constrained continuous-time systems with cone properties. Automatic Control, IEEE Transactions on, 39(2):401-405, Feb 1994.

[41] A. Tiwari, J. Fung, Bhattacharya R., and Murray R. M. Polyhedral cone invariance applied to rendezvous of multiple agents. In Decision and Control, 2004. CDC. 43rd IEEE Conference on, volume 1, pages 165-170 Vol.1, 2004.

[42] Vincent A. Traag, Yurii E. Nesterov, and Paul Van Dooren. Exponential ranking: taking into account negative links. In Proceedings of the Second international conference on Social informatics, SocInfo'10, pages 192 202, Berlin, Heidelberg, 2010. Springer-Verlag.

[43] M. Valcher and L. Farina. An algebraic approach to the construction of polyhedral invariant cones. SIAM Journal on Matrix Analysis and Applications, 22(2):453-471, 2000.

[44] S. Wasserman and K. Faust. Social Network Analysis: methods and applications. Cambridge Univ. Press, 1994.

[45] Weiguo Xia and Ming Cao. Clustering in diffusively coupled networks. Automatica, 47(11):2395 - 2405, 2011.

[46] Yuecheng Yang, D.V. Dimarogonas, and Xiaoming Hu. Opinion consensus of modified Hegselmann-Krause models. In Decision and Control (CDC), 2012 IEEE 51st Annual Conference on, pages 100-105, 2012.

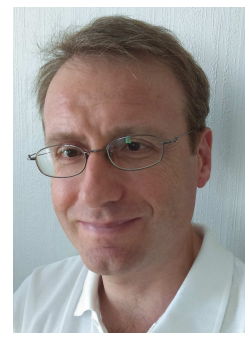

Claudio Altafini received a Master degree ("Laurea") in Electrical Engineering from the University of Padova, Italy, in 1996 and a PhD in Optimization and Systems Theory from the Royal Institute of Technology, Stockholm, Sweden in 2001. From 2001 till 2013 he was with the International School for Advanced Studies (SISSA) in Trieste, Italy. Since 2014 he is a Professor in the Division of Automatic Control, Dept. of Electrical Engineering at Linköping University, Sweden. He is currently serving as an Associate Editor for the IEEE Trans. on Automatic Control. His research interests are in the areas of nonlinear systems analysis and control, with applications to quantum mechanics, systems biology and complex networks.

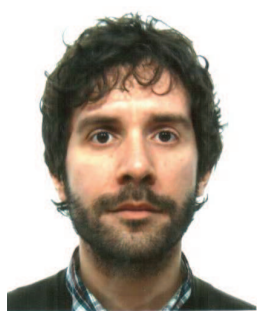

Gabriele Lini is Control System Designer for ADAS systems at the CTO of Magneti Marelli SpA, Turin, Italy. He is also responsible of a research center at the University of Parma, born from the cooperation between Magneti Marelli and VisLab. In 2012-13, he was a PostDoc Researcher at the International School for Advanced Studies (SISSA) of Trieste, Italy, and also cooperated with Glance Vision Technologies srl, Trieste. From 2009 until 2012, he was a PhD student at Dipartimento di Ingegneria dell'Informazione at the University of Parma, Italy. In 2009 he was a visiting scholar at the University of Toronto, Canada. In 2008 he obtained a laurea cum laude in electronic and computer engineering at the University of Parma. His main research topics are timeoptimal control, dynamic inversion for nonlinear systems, feedforwar/feedback control strategies, path planning, trajectory tracking, path following, consensus theory, linear algebra and artificial vision. 